\title{
The Solution of Linear Inverse Problems in Satellite Geodesy by Means of Spherical Spline Approximation
}

\author{
$b y$ \\ F. SCHNEIDER \\ University of Kaiserslautern \\ Laboratory of Technomathematics \\ Geomathematics Group \\ 67663 Kaiserslautern \\ Germany
}

\begin{abstract}
In this paper we consider a certain class of geodetic linear inverse problems $\Lambda F=G$ in a reproducing kernel Hilbert space setting to obtain a bounded generalized inverse operator $\Lambda^{\dagger}$. For a numerical realization we assume $G$ to be given at a finite number of discrete points to which we employ a spherical spline interpolation method adapted to the Hilbert spaces. By applying $\Lambda^{\dagger}$ to the obtained spline interpolant we get an approximation of the solution $F$. Finally our main task is to show some properties of the approximated solution and to prove convergence results if the data set increases.
\end{abstract}

\section{Introduction}

Until the fifties the essential part of gravitational potential determination was obtained by terrestrial observations. In 1957 the situation changed completely with the first satellite mission. Since that time the mathematical techniques of gravitational potential determination had to be adapted to the new situation. The arising problem was to determine the potential on and outside the sirface of the earth from discretely given measurements at satellite altitude which, in addition, could be of different type depending on the mission (e.g. satellite-to-satellite tracking (SST) or gradiometry cf. Rummel(1979) and Rummel(1986)). This so called downward continuation process is formulated as an inverse problem in terms of an integral operator equation which is severely ill conditioned. Therefore several approaches of regularization methods are in discussion. Thalhammer (1995) proposes a discretization of the (linear) operator using projection methods for regularization and the minimization of a Tikhonov functional, while Schreiner(1994) considers a locally supported spline approximation of the given data and a regularization by a truncated series expansion of the generalized operator. Stochastic methods are discussed e.g. in Rummel(1979) and a boundary vahe approach is given in Keller and Hirsch(1994). Our 1

"Submited to "Manuscripta Geodaetica" 
solution is situated in a reproducing kernel Hilbert space setting (see Nashed and Wahba(1974) and Wahba(1973)) using a spherical spline interpolation method (see Freeden(1990)), where the regularization of the inverse problem is done by refinement of the topologies. In the first part of our work we assume spherical shape for all surfaces but the main results of this paper can be found in Section 6, where the attention is directed to the fact that the satellite orbit as well as the earth's surface do not underly severe geometrical restrictions. In what follows, we summarize briefly the outline of this paper.

In Section 2 we give an overview of the notation, definitions and mathematical structures we need. Section 3 deals with the formulation of the type of inverse problems (in a spherical context) we discuss. Moreover, the connection to certain satellite missions is demonstrated. After that we deal with regularization which leads to the introduction of the reproducing kernel Hilbert spaces adapted to the inverse problem in Section 4. Then we proceed with the definition of spherical spline interpolation in Section 5. Here we assume the data to be error free and develop some minimal properties of the solution and convergence statements. After that we consider error affected data and formulate a combined interpolation and smoothing method. Finally, in Section 6 we generalize the whole problem to non-spherical structures in the following sense. The satellite orbit is no longer assumed to be spherical as well as the surface of the earth. Then we calculate an approximation of the gravitational potential on the surface of the earth from given data on the satellite orbit. At last we show again some minimal properties of the approximation and a convergence result if the measurements at the orbit increase.

\section{Preliminaries}

For any $x=\left(x_{1}, x_{2}, x_{3}\right)^{T} \in \mathbb{R}^{3}$ different from the origin we may write $x=r \xi$, where $|x|=r$ and $\xi \in \Omega_{1}=\left\{x \in \mathbb{R}^{3}|| x \mid=1\right\}$ (unit sphere). To be consistent we define $\Omega_{R}=\left\{x \in \mathbb{R}^{3}|| x \mid=R\right\}$ to be the sphere with radius $R>0$ (centered at the origin). The canonical basis of $\mathbb{R}^{3}$ is denoted by $\left\{\varepsilon^{1}, \varepsilon^{2}, \varepsilon^{3}\right\}$. As usual, $\Delta_{\xi}^{*}$ denotes the Beltrami operator. It is well known that the only eigenfunctions of $\Delta_{\xi}^{*}$ are the spherical harmonics $Y_{n, j}: \Omega_{1} \rightarrow \mathbb{R}, \Delta_{\xi}^{*} Y_{n, j}--n(n+1) Y_{n, j} ; n=$ $0,1, \ldots, j=1, \ldots, 2 n+1$, where $2 n+1$ is the number of linearly independent elements of order $n$. The Legendre polynomials $P_{n}:[-1,1] \rightarrow \mathbb{R}$ are the only everywhere on $[-1,1]$ infinitely often differentiable eigenfunctions of the Legendre operator $\left(1-t^{2}\right)\left(\frac{d}{d t}\right)^{2}-2 t\left(\frac{d}{d t}\right)$ which satisfy $P_{n}(1)=1$. If we consider $P_{n}\left(\varepsilon^{3} \cdot\right): \Omega_{1} \rightarrow \mathbb{R}$ one can show (cf. Müller(1966)) that $P_{n}\left(\varepsilon^{3} \cdot\right)$ is the only spherical harmonic that is invariant under orthogonal transformations which leave $\varepsilon^{3}$ fixed. The comnection between $P_{n}\left(\varepsilon^{3} \cdot\right)$ and an $\mathcal{L}^{2}\left(\Omega_{1}\right)$-orthonormalized set of spherical harmonics $\left\{Y_{n, j}\right\}: j=1 \ldots 2 n+1$ (from now on always assumed) is stated in the addition theorem (cf. Müller(1966) and Freeden(1979))

$$
\sum_{j=1}^{2 n+1} Y_{n, j}(\xi) Y_{n, j}(\eta)=\frac{2 n+1}{4 \pi} P_{n}(\xi \eta)
$$

For the following considerations we need some Fourier analysis on $\mathcal{L}^{2}$-spaces. Therefore we define as usual

$$
\mathcal{L}^{2}\left(\Omega_{R}\right)=\left\{F: \Omega \rightarrow \mathbb{R} \mid F \text { measurable, }\left\|F^{\prime}\right\|_{L^{2}\left(\Omega_{R}\right)}^{2}<\infty\right\}
$$


with

$$
\left\|F^{\prime \prime 2}\right\|_{\left(\Omega_{R}\right)}=\left(\int_{\Omega_{R}}|F(y)|^{2} d \omega_{R}(y)\right)^{\frac{1}{2}}
$$

The class of functions on $\Omega_{R}$ which are k-times continnously differentiable is denoted by $\mathcal{C}^{k}\left(\Omega_{R}\right)$, where $C^{0}\left(\Omega_{R}\right)=\mathcal{C}\left(\Omega_{R}\right)$ and

$$
\|F\|_{\mathcal{C}\left(\Omega_{R}\right)}=\sup _{y \in \Omega_{R}}|F(y)|
$$

As it is well known that $\left\{Y_{n, j}\right\}: n=0,1, \ldots, j=1, \ldots, 2 n+1$ forms a complete and closed system in $L^{2}\left(\Omega_{1}\right)$ we can represent any $F \in \mathcal{L}^{2}\left(\Omega_{1}\right)$ in terms of spherical harmonics

$$
F(\xi)=\sum_{n=0}^{\infty} \sum_{j=1}^{2 n+1} F_{n, j} Y_{n, j}(\xi)
$$

with

$$
F_{n, j}=\int_{\Omega_{1}} F(\xi) Y_{n, j}(\xi) d \omega(\xi)
$$

being the Fourier coefficients of $F$. To translate this concept to $\mathcal{L}^{2}\left(\Omega_{R}\right)$ we introduce

$$
Y_{n, j}^{R}(y)=\frac{1}{R} Y_{n, j}\left(\frac{y}{|y|}\right) ; y \in \Omega_{R}
$$

such that $\left\{Y_{n, j}^{R}\right\} ; n=0,1 \ldots j=1 \ldots 2 n+1$ forms a complete and closed system in $\mathcal{L}^{2}\left(\Omega_{R}\right)$ which is orthonormal in the $(\cdot,)_{\mathcal{L}^{2}\left(\Omega_{R}\right)^{- \text {sense. }}}$ Consequently any function $F \in \mathcal{L}^{2}\left(\Omega_{R}\right)$ can be represented as

$$
F(y)=\sum_{n=0}^{\infty} \sum_{j=1}^{2 n+1} F_{n, j}^{R} Y_{n, j}^{R}(y)
$$

with

$$
F_{n, j}^{R}=\int_{\Omega_{R}} F(y) Y_{n, j}^{n}(y) d \omega_{R}(y)
$$

As we shall see later the theory of reproducing kernel Hilbert structure in Sobolev spaces is essential for spherical spline interpolation (cf. Freeden(1990)). For that purpose we want to give a short introduction here which is deepened in Section 4 . Let $\left\{A_{n, j}\right\} ; n=0,1, \ldots, j=1, \ldots, 2 n+1$, denote a sequence of real numbers, where $\left\{A_{n}\right\} ; n=0,1, \ldots$ is understood to be equivalent to $\left\{A_{n, j}\right\}$ with $A_{n, j}=A_{n}: j=1, \ldots 2 n+1$. Considering the space

$$
\varepsilon\left(\left\{A_{n}\right\}\right)=\left\{\left.F \in C^{\infty}\left(\Omega_{R}\right)\left|\sum_{n=0}^{\infty} \sum_{j=1}^{2 n+1}\right| A_{n}\right|^{2}\left(F_{n, j}^{R}\right)^{2}<\infty\right\}
$$

we are able to define an inner product

$$
\left(F .(r)_{H\left(\left\{A_{n}\right\} \Omega_{R}\right)}=\sum_{n=0}^{\infty} \sum_{n=1}^{2 n+1}\left|A_{n}\right|^{2} F_{n, j}^{R} G_{n, j}^{R}:\right.
$$

$F . G \in E\left(\left\{A_{n}\right\}\right)$ and the associated norm

$$
\|F\|_{M\left(\left\{A_{n}\right\} \Omega_{R}\right)}=\left(F F_{h\left(\left\{A_{n}\right\}, \Omega_{R}\right\}}\right)^{\frac{1}{2}}
$$


$F \in \mathcal{E}\left(\left\{A_{n}\right\}\right)$ on $\mathcal{E}\left(\left\{A_{n}\right\}\right)$. Then the Sobolev space $\mathcal{H}\left(\left\{A_{n}\right\}, \Omega_{R}\right)$ is the completion of $\mathcal{E}\left(\left\{A_{n}\right\}\right)$ under the norm (4). $\mathcal{H}\left(\left\{A_{n}\right\}, \Omega_{R}\right)$ equipped with the inner product (3) is a Hilbert space. If we consider $A_{n}=\left(n+\frac{1}{2}\right)^{s}$ we define $\mathcal{H}_{s}\left(\Omega_{R}\right)=\mathcal{H}\left(\left\{A_{n}\right\}, \Omega_{R}\right)$. In particular, $\mathcal{H}_{0}\left(\Omega_{R}\right)=\mathcal{L}^{2}\left(\Omega_{R}\right)$ and $\mathcal{H}_{s}\left(\Omega_{R}\right) \subset \mathcal{H}_{t}\left(\Omega_{R}\right)$ for $t<s$ with $\|F\|_{\mathcal{H}_{t}\left(\Omega_{R}\right)} \leq\|F\|_{\mathcal{H}_{3}\left(\Omega_{R}\right)}$. If we furthermore introduce the Beltrami operator $\Delta_{y}^{*}, R$ of the sphere $\Omega_{R}$, given by $\Delta_{y}^{*, R}=\frac{1}{R^{2}} \Delta_{\frac{y}{|y|}}^{*}$ we have

$$
\|F\|_{\mathcal{H}_{3}\left(\Omega_{R}\right)}=\left\|\left(-R^{2} \Delta_{y}^{*, R}+\frac{1}{4}\right) F\right\|_{\mathcal{L}^{2}\left(\Omega_{R}\right)}
$$

and

$$
\left\|\left(-R^{2} \Delta_{y}^{*, R}+\frac{1}{4}\right)^{(t / 2)} F\right\|_{\mathcal{H}_{s}\left(\Omega_{R}\right)}=\|F\|_{\mathcal{H}_{s+t}\left(\Omega_{R}\right)}
$$

for all $F \in \mathcal{H}_{s}\left(\Omega_{R}\right)$.

Now we are in a position to turn over to the last part of this section. In order to classify the type of inverse problems we deal with, it is nescessary to introduce spherical pseudodifferential operators (SPDO) (cf. Èskin(1981), Svensson(1983)). Following Cui and Freeden(1995) we consider a sequence of real numbers $\left\{\Lambda^{\wedge}(n)\right\} ; n=0,1, \ldots$ satisfying

$$
\lim _{n \rightarrow \infty} \frac{\left|\Lambda^{\wedge}(n)\right|}{\left(n+\frac{1}{2}\right)^{t}}=\text { consl } \neq 0
$$

for some $t \in \mathbb{R}$. Then the operator $\Lambda: \mathcal{H}_{s}\left(\Omega_{R}\right) \mapsto \mathcal{H}_{s-t}\left(\Omega_{R}\right)$ defined by

$$
\Lambda F=\sum_{n=0}^{\infty} \sum_{j=1}^{2 n+1} \Lambda^{\wedge}(n) F_{n, j}^{R} Y_{n, j}^{R} ; \quad F \in \mathcal{H}_{s}\left(\Omega_{R}\right)
$$

is called SPDO of order $t$, where $\left\{\Lambda^{\wedge}(n)\right\}$ is called (spherical) symbol of $\Lambda$ and the convergence of the series is understood in the $\mathcal{H}_{s-t}\left(\Omega_{R}\right)$ topology. Moreover, if

$$
\lim _{n \rightarrow \infty} \frac{\left|\Lambda^{\wedge}(n)\right|}{\left(n+\frac{1}{2}\right)^{t}}=0
$$

for all $t \in \mathbb{R}$ then the operator $\Lambda: \mathcal{H}_{s}\left(\Omega_{R}\right) \mapsto \mathcal{C}^{\infty}\left(\Omega_{R}\right)$ is called SPDO of order $-\infty$. If in addition

$$
\sum_{n=0}^{\infty} \frac{2 n+1}{4 \pi} \Lambda^{\wedge}(n)<\infty
$$

we define

$$
K_{\Lambda}(\tilde{y}, y)=\sum_{n=0}^{\infty} \sum_{j=1}^{2 n+1} \Lambda^{\wedge}(n) Y_{n, j}^{R}(\tilde{y}) Y_{n, j}^{R}(y)
$$

to be the kernel of $\Lambda$ such that

$$
\Lambda F=\int_{\Omega_{R}} K_{\Lambda}(\cdot, y) F(y) d \omega_{R}(y)
$$

Some properties of $\Lambda$ and $\Lambda^{\wedge}(n)$ are simply verified: $\left(\Lambda^{\prime}+\Lambda^{\prime \prime}\right)^{\wedge}(n)=\left(\Lambda^{\prime}\right)^{\wedge}(n)+\left(\Lambda^{\prime \prime}\right)^{\wedge}(n)$, $\left(\Lambda^{\prime} \Lambda^{\prime \prime}\right)^{\wedge}(n)=\left(\Lambda^{\prime}\right)^{\wedge}(n)\left(\Lambda^{\prime \prime}\right)^{\wedge}(n)$ for all $n=0,1, \ldots$ and $\Lambda Y_{n, j}^{R}=\Lambda^{\wedge}(n) Y_{n, j}^{R} ; n=0,1, \ldots$, $j=1, \ldots, 2 n+1$. 


\section{The Inverse Problem}

Let $\left\{\Lambda^{\wedge}(n)\right\}$ with $\Lambda^{\wedge}(n)>0$ for all $n=0,1, \ldots$ denote the symbol of a SPDO of order $t \in[0, \infty)$, i.e. for any $G \in \mathcal{H}_{s}\left(\Omega_{r}\right)$ we have

$$
(\Lambda G)(x)=\sum_{n=0}^{\infty} \sum_{j=1}^{2 n+1} \Lambda^{\wedge}(n) G_{n, j}^{r} Y_{n, j}^{r}(x) \in H_{s-1}\left(\Omega_{,}\right) .
$$

Furthermore.

$$
A_{\frac{R}{r}}(x, y)=\sum_{n=0}^{\infty} \sum_{j=1}^{2 n+1}\left(\frac{R}{r}\right)^{n} Y_{n, j}^{r}(x) Y_{n, j}^{R}(y)
$$

$x=r \xi, y=R \eta, R<r$ defines the kernel of a SPDO $A_{\frac{R}{r}}: L^{2}\left(\Omega_{R}\right) \rightarrow C^{\infty}\left(\Omega_{r}\right)$,

$$
\left(A_{R} F\right)(x)=\int_{\Omega_{R}} F(y) A_{\frac{R}{r}}(x, y) d \omega_{R}(y)=\sum_{n=0}^{\infty} \sum_{j=1}^{2 n+1}\left(\frac{R}{r}\right)^{n} F_{n, j}^{R} Y_{n, j}^{r}(x)
$$

of order $-\infty$. Combining both SPDO's we obtain:

$\Lambda_{\frac{R}{r}}: \mathcal{L}^{2}\left(\Omega_{R}\right)+\mathcal{C}^{\infty}\left(\Omega_{r}\right)$ given by

$$
\left(\Lambda_{\frac{R}{r}} F\right)(x)=\int_{\Omega_{R}} F(y) \Lambda_{\Lambda_{\frac{R}{r}}}(x, y) d \omega_{R}(y)=\sum_{n=0}^{\infty} \sum_{j=1}^{2 n+1}\left(\frac{R}{r}\right)^{n} \Lambda^{\wedge}(n) F_{n, j}^{R} Y_{n, j}^{r}(x)
$$

is an injective SPDO of order $-\infty$ with symbol $\left\{\left(\frac{R}{r}\right)^{n} \Lambda^{\wedge}(n)\right\}$ having the kernel

$$
K_{\Lambda_{\frac{R}{1}}}(x, y)=\sum_{n=0}^{\infty} \sum_{j=1}^{2 n+1}\left(\frac{R}{r}\right)^{n} \Lambda^{\wedge}(n) Y_{n, j}^{r}(x) Y_{n, j}^{R}(y)
$$

$x \in \Omega_{r}, y \in \Omega_{R}$. This operator defines the class of inverse problems we want to discuss. Given a function $G \in \mathcal{L}^{2}\left(\Omega_{r}\right)$ we are interested in solving the inverse problem

$$
\Lambda_{\frac{R}{r}} F=G: \quad F \in \mathcal{L}^{2}\left(\Omega_{R}\right) .
$$

$\Lambda_{\frac{R}{r}}$ as defined in (5) with kernel $K_{\Lambda_{\frac{R}{r}}}$ defined in (6). Inverse problems of type ( 7 ) are known as Fredholm integral equations of the first kind. The generalized inverse of the SPDO $\Lambda_{\frac{R}{r}}$ denoted by $\Lambda_{\frac{R}{r}}^{\dagger}$ is an operator $I_{\frac{R}{r}}^{\dagger}: D\left(\Lambda_{\frac{R}{T}}^{\dagger}\right) \subset L^{2}\left(\Omega_{p}\right)-\mathcal{L}^{2}\left(\Omega_{R}\right) . G-\Lambda_{\frac{R}{r}}^{\dagger} G=F$, where $F$ is uniquely defined by $\Lambda_{\frac{R}{r}} F=P \overline{R\left(\Lambda_{\left.\frac{R}{r}\right)}\right.} G \cdot D\left(\Lambda_{\frac{R}{r}}\right)$ defines the domain of $\Lambda_{\frac{R}{r}}^{+}$and $P_{\overline{R\left(\Lambda_{\frac{R}{F}}\right.}} G$ denotes the orthogonal projection of any function $G \in \mathcal{L}^{2}\left(\Omega_{r}\right)$ onto the closed range of $\Lambda_{\frac{R}{r}}$ (where we used the same notation as in Lonis(1989)). Due to the fact that in our case $\Lambda_{\frac{R}{r}}^{r}$ represents a compact operator on $L^{2}\left(\Omega_{R}\right)$ (as $\left.K_{\frac{R}{r}} \in \mathcal{C}\left(\Omega_{*} \times \Omega_{R}\right)\right)$ with infinitely dimensional range in $L^{2}\left(\Omega_{r}\right)$ the generalized inverse operator $\lambda_{R}$ is not bounded (cf. Nashed and Wahba(1974)). The kind of ill-posedness is easy to see by calculating the singular system of (7). as $\Lambda_{\frac{R}{r}} Y_{n, j}^{R}=\sigma_{n} Y_{n, j}^{r}$ 
and $\Lambda_{\underline{R}}^{*} Y_{n, j}^{r}=\sigma_{n} Y_{n, j}^{R}$ with $\sigma_{n}=\left(\frac{R}{r}\right)^{n} \Lambda^{\wedge}(n)$ (i.e. $\sigma_{n}^{-1}=\left(\frac{r}{R}\right)^{n} \Lambda^{\wedge}(n)^{-1}$ and $\frac{r}{R}>1$ ), where $\Lambda_{\frac{R}{r}}^{*}: \mathcal{L}^{2}\left(\Omega_{r}\right) \rightarrow \mathcal{C}^{\infty}\left(\Omega_{R}\right)$ denotes the adjoint operatcr. With this it follows immediately that the inverse problem (7) is exponentially ill-posed (cf. Louis(1989)) and the regularization technique we want to apply is presented in Section 4. Finally, we would like to emphasize the importance of equation (7) in many problems of satellite geodesy:

Assuming the spherical shape of the earth and the satellite orbit (what we do no longer require in Section 6), equation (7) enables us to recover the following satellite problems. If we define $R$ : radius of the earth, $H$ : satellite altitude, $r=R+H$ and $F$ : gravitational potential on the surface of the earth, we have:

\section{The Linearized SST-Problem}

$\Lambda^{\wedge}(n)=\frac{n+1}{r}, C_{r}$ negative radial derivative of the gravitational potential at the satellite orbit

\section{The Gradiometry Problem}

$\Lambda^{\wedge}(n)=\frac{(n+1)(n+2)}{r^{2}}, G 2$ nd radial derivative of the gravitational potential at the satellite orbit

\section{The Regularization}

Following the ideas of Nashed and Wahba(1974) we consider the inverse problem ( 7 ) in Hilberttype subspaces of $\mathcal{L}^{2}\left(\Omega_{R}\right)$ and $\mathcal{L}^{2}\left(\Omega_{r}\right)$, respectively, in order to obtain a bounded generalized inverse operator which is known as regularization by refinement of the topologies (cf. e.g. Louis(1989)). Therefore it is necessary to extend the knowledge about the theory of Sobolev spaces already introduced in Section 2.

According to Freeden( 1990 ) we call a sequence $\left\{A_{n}\right\}$ of real numbers summable if

1. $A_{n} \neq 0 ; n=0,1, \ldots$

2. $\sum_{n=0}^{\infty} \frac{2 n+1}{4 \pi} \frac{1}{A_{n}^{2}}<\infty$.

Starting with a summable sequence $\left\{\Lambda_{n}\right\}$, the Sobolev space

$$
\mathcal{H}\left(\left\{A_{n}\right\} . \Omega_{R}\right)=\frac{\left\{\left.F \in C^{\infty}\left(\Omega_{R}\right)\left|\sum_{n=0}^{\infty} \sum_{j=1}^{2 n+1}\right| A_{n}\right|^{2}\left(F_{n, j}^{R}\right)^{2}<\infty\right\}}{\|\cdot\|_{\mathcal{H}\left(\left\{A_{n}\right\}, \Omega_{R}\right)}}
$$

is a separable Hilbert space (with Hilbert basis $\left\{\frac{1}{A_{n}} Y_{n, j}^{R}\right\}$ ) and inner product defined in (3) possessing the reproducing kernel

$$
K_{H\left(\left\{A_{n}\right\} \Omega_{R}\right)}(\tilde{y}, y)=\sum_{n=0}^{\infty} \sum_{j=1}^{2 n+1} \frac{1}{\left|A_{n}\right|^{2}} Y_{n, j}^{R}(\tilde{y}) Y_{n, j}^{R}(y) ;
$$

$\tilde{y}, y \in \Omega_{R}$ (cf. Freeden(1990) and Aronszajn(1950)), i.e. 
1. for fixed $\left.\ddot{y} \in \Omega_{R}: \quad L_{\mathcal{H}}\left(A_{n}\right\}, \Omega_{R}\right)\left(\breve{y}_{\cdot}\right)$ is of class $\mathcal{H}\left(\left\{A_{n}\right\}, \Omega_{R}\right)$

2. $\left(K_{\mathcal{H}\left(\left\{A_{n}\right\}, \Omega_{R}\right)}(\tilde{y} \cdot \cdot), F\right)_{\mathcal{H}\left(\left\{A_{n}\right\}, \Omega_{R}\right)}=F(\tilde{y})$ for all $F \in \mathcal{H}\left(\left\{A_{n}\right\}, \Omega_{R}\right)$.

As we want to work in the inverse problem context we have to choose those sequences such that we obtain a reproducing kernel Hilbert-type subspace of $L^{2}\left(\Omega_{R}\right)$ as well as a Hilbert-type subspace of $L^{2}\left(\Omega_{r}\right)$ and moreover that the generalized inverse operator is bounded on the latter one. Firstly, we prove

Lemma 1 Let $f: \mathbb{R}^{+} \rightarrow \mathbb{R}^{+}$be a function such that the sequence $\left\{\left(f\left(\sigma_{n}\right)\left(\frac{r}{R}\right)^{2 n}\right)^{-\omega^{\frac{1}{2}}}\right\}$ with $\sigma_{n}=\left(\frac{R}{r}\right)^{n} \Lambda^{\wedge}(n)$ is summable. Furthermore, we define the sequences $\left\{A_{n}^{R}\right\}=\left\{\left(f\left(\sigma_{n}\right) \sigma_{n}^{-2}\right)^{-\frac{1}{2}}\right\}$, $\left\{B_{n}^{r}\right\}=\left\{\left(f\left(\sigma_{n}\right)\right)^{-\frac{1}{2}}\right\}$ and $\left\{\tilde{A}_{n}\right\}=\left\{\left(f\left(\sigma_{n}\right) \sigma_{n}{ }^{1} \frac{R}{r}\right)^{-\frac{1}{2}}\right\}$. Then $\left\{A_{n}^{R}\right\} \cdot\left\{B_{n}^{r}\right\}$ and $\left\{\tilde{A}_{n}\right\}$ are summable.

\section{Proof:}

$$
\sum_{n=0}^{\infty} \frac{2 n+1}{4 \pi} \frac{1}{\left(A_{n i}^{12}\right)^{2}}=\sum_{n=0}^{\infty} \frac{2 n+1}{4 \pi} f\left(\sigma_{n}\right) \sigma_{n}^{-2}=\sum_{n=0}^{\infty} \frac{2 n+1}{4 \pi} f\left(\sigma_{n}\right)\left(\frac{r}{R}\right)^{2 n}\left(\Lambda^{\wedge}(n)\right)^{-2}<\infty
$$

because of the requirements on $f$ and the fact that $\Lambda$ is a SPDO of order $t \geq 0$. Thus, $\left\{A_{n}^{R}\right\}$ is summable. The summability of $\left\{B_{n}^{r}\right\}$ and $\left\{\tilde{A}_{n}\right\}$ follows immediately as $\lim _{n \rightarrow \infty} \sigma_{n}=0$.

Hence, we immedeately obtain

Theorem 1 If the corresponding Hilbert spaces are denoted by $\mathcal{l}^{R}=\eta\left(u\left(A_{n}^{R}\right\}, \Omega_{R}\right), \mathcal{T}^{r}-$ $\mathcal{H}\left(\left\{B_{n}^{r}\right\}, \Omega_{r}\right)$ and $\widetilde{\mathcal{H}}=\mathcal{H}\left(\left\{\tilde{A}_{n}\right\}, \Omega_{R}\right)$ with reproducing kernels $K_{H_{R}}(\cdot, \cdot), K_{\mathcal{H}^{r}}(\cdot, \cdot)$ and $K_{\widetilde{\mathcal{H}}}(\cdot, \cdot)$, respectively, the following statements are valid:

1. $\left(\Lambda_{\frac{R}{r}} K_{\tilde{H}}(\tilde{y} \cdot)\right)(x)=K_{H}(\tilde{x}, x) ; \quad \ddot{y} \in \Omega_{R}, \tilde{x} \in \Omega_{r}$ with $\tilde{y}=R \tilde{\eta} . \tilde{x}=r \ddot{\eta}, \ddot{\eta} \in \Omega_{1}$.

2. $\Lambda_{\frac{B}{r}}\left(\mathcal{H}^{R}\right) \subseteq \mathcal{H}^{r}$ and $\Lambda_{\frac{R}{r}}(\tilde{H}) \subseteq \mathcal{H}^{r}$.

\section{Proof:}

ad 1)

$\left(\lambda_{\frac{R}{r}} h_{r}(y \cdot y)(x)\right.$

$$
\begin{aligned}
& =\int_{\Omega_{R}}\left(\sum_{n=0}^{x} \sum_{j=1}^{2 n+1} \sigma_{n, j} Y_{n, j}^{R}(y) Y_{n, j}^{r}(x)\right)\left(\sum_{n=0}^{\infty} \sum_{j=1}^{2 n+1} \frac{R}{r} f\left(\sigma_{n}\right) \sigma_{n}^{-1} Y_{n, j}^{R}(y) Y_{n, j}^{R}(y)\right) d \omega_{R}(y) \\
& =\sum_{n=0}^{x} \sum_{j=1}^{2 n+1} f\left(\sigma_{n j}\right) Y_{n, j}(r) Y_{n, j}(x) \\
& =h_{H}(\tilde{x}, x)
\end{aligned}
$$


ad 2)

An immediate consequence of Lemma 1 is the fact that $\mathcal{H}^{R} \subset \mathcal{C}\left(\Omega_{R}\right), \widetilde{\mathcal{H}} \subset \mathcal{C}\left(\Omega_{R}\right)$ and $\mathcal{H}^{r} \subset \mathcal{C}\left(\Omega_{r}\right)$ (cf. Freeden $(1990)$ ). If we consider a set $X_{\infty}^{1}=\left\{\eta_{k}\right\}$ of pairwisely distinct elements $\eta_{k} \in \Omega_{1}$ such that $X_{\infty}^{1}$ is dense in $\Omega_{1}$ (with respect to the Euclidean topology) it is clear that $X_{\infty}^{R}=$ $\left\{y_{k}\right\}, y_{k}=R \eta_{k}$ is dense in $\Omega_{R}$. Then it is well known (cf. Meschkowski(1962)) that $\left\{K_{\mathcal{H}^{R}}\left(y_{k}, \cdot\right)\right\}$ forms a complete and closed system in $\mathcal{H}^{R}$ (with respect to $\|\cdot\|_{\mathcal{H}^{R}}$ ). Thus, we only have to show that $\Lambda_{\frac{R}{r}} K_{\mathcal{H}^{R}}(y, \cdot) \in \mathcal{H}^{r}$ and $\left.\Lambda_{\frac{R}{r}} K_{\widetilde{\mathcal{H}}^{(y,}} \cdot\right) \in \mathcal{H}^{r}$ which can be done by an easy calculation.

Now we are in a position to reformulate the inverse problem $(7)$ in refined topologies. Given a function $G \in \mathcal{H}^{r} \subset \mathcal{C}\left(\Omega_{r}\right) \subset \mathcal{L}^{2}\left(\Omega_{r}\right)$ we search for a solution of the problem

$$
\Lambda_{\frac{R}{r}} F=G ; \quad F \in \mathcal{H}^{R} .
$$

In order to show the well-posedness of (8) we prove

Theorem 2 Let $X_{\infty}^{R}=\left\{y_{k}\right\}, y_{k}=R \eta_{k}$ denote a dense subset of $\Omega_{k}$ (of pairwisely distinct ele-

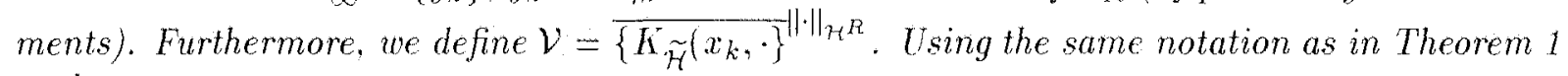
we have

1. $\tilde{\mathcal{H}} \subseteq \mathcal{H}^{R}$

2. $\left(\mathcal{V},\|\cdot\|_{\mathcal{H}^{R}}\right) \stackrel{\Lambda_{\frac{R}{r}}}{\longrightarrow}\left(\mathcal{H}^{r},\|\cdot\|_{\mathcal{H}^{r}}\right)$ is isometrically isomorphic

3. $\mathcal{V}=\mathcal{H}^{R}$

4. $\left(\mathcal{H}^{R},\|\cdot\|_{\mathcal{H}^{R}}\right) \stackrel{\Lambda_{\frac{R}{r}}}{\longrightarrow}\left(\mathcal{H}^{r},\|\cdot\|_{\mathcal{H}^{r}}\right)$ is isometrically isomorphic

\section{Proof:}

ad 1)

As before it is easy to show that $K_{\widetilde{\mathcal{H}}}(\cdot, \cdot) \in \mathcal{H}^{R}$.

ad 2)

In Theorem 1 we have already shown that $\left(\Lambda_{\underline{R}} K_{\tilde{\mathcal{H}}}(\tilde{y}, \cdot)\right)(x)=K_{\mathcal{H}}(\tilde{x}, x)$. Using 1$)$ we consider the closure of $\left\{K_{\tilde{\mathcal{H}}^{*}}\left(y_{k}, \cdot\right)\right\}$ in $\mathcal{H}^{R}$ with respect to $\|\cdot\|_{\mathcal{H}^{R}}$ (denoted by $\mathcal{V}$ ). Moreover, we obtain

$$
\begin{aligned}
\|F\|_{\mathcal{H}^{n}}^{2} & =\sum_{n=0}^{\infty} \sum_{j=1}^{2 n+1}\left(f\left(\sigma_{n}\right)\right)^{-1} \sigma_{n}^{2}\left(F_{n, j}^{R}\right)^{2} \\
& =\sum_{n=0}^{\infty} \sum_{j=1}^{2 n+1}\left(f\left(\sigma_{n}\right)\right)^{-1}\left(\sum_{n=0}^{\infty} \sum_{j=1}^{2 n+1} \sigma_{n} Y_{n, j}^{r}(x) F_{n, j}^{R}, Y_{n, j}^{r}(x)\right)_{\mathcal{L}^{2}\left(\Omega_{r}\right)}^{2} \\
& =\left\|\Lambda_{\frac{R}{r}} F\right\|_{\mathcal{H}^{r}}^{2} .
\end{aligned}
$$

Then it is not difficult to see that

$$
\Lambda_{\frac{R}{r}}(\mathcal{V})=\Lambda_{\frac{R}{r}}\left(\overline{\left\{\Lambda_{\tilde{H}}\left(y_{k}, \cdot\right)\right\}^{-1} \|_{H_{R} R}}\right)=H^{r} .
$$


Thus, the generalized inverse operator $\Lambda_{\frac{R}{r}\left(\mathcal{H}^{r}, \mathcal{H}^{*}\right)}^{\dagger}: \mathcal{H}^{r}-\mathcal{V}$ is bonnded as the image of $\mathcal{V}$ is closed in ' $\mathcal{H}^{r}$. where $\Lambda_{\frac{R}{r},\left(H^{*}, H^{R}\right)}^{\dagger}$ is understood to be the restriction of the generalized inverse operator $\Lambda_{R}^{\dagger}$ onto $\mathcal{H}^{r}$. So there is a one-to-one correspondence between a function $F \in \mathcal{V}$ and a function $G \in \mathcal{H}^{r}$ via $\Lambda_{\frac{R}{r}} F=G$ and $F=\Lambda_{\frac{R}{r}\left(\mathcal{H}^{r} \cdot \mathcal{H}^{H}\right)}^{\dagger}($.

ad 3$)$

As $\mathcal{V} \subseteq \mathcal{H}^{R}$ we can decompose $\mathcal{H}^{R}$ into $\mathcal{H}^{R}=\mathcal{V} \oplus \mathcal{V}^{\perp}\left(\perp\right.$ in the $\mathcal{H}^{R}$-sense). Then we know that $\overline{\mathcal{H}^{r}}=\Lambda_{\underline{R}}(\mathcal{V}) \subseteq \Lambda_{\underline{R}}\left(\mathcal{H}^{R}\right) \subseteq \mathcal{H}^{r}$. Thus, $\Lambda_{\frac{R}{r}}(\mathcal{V})=\Lambda_{\underline{R}}\left(\mathcal{H}^{R}\right)$ and $\mathcal{V}^{\perp}$ is the nullspace of the operator $\Lambda_{\frac{R}{N}}$ in $H^{R}$ which is always $\{0\}$ for the class of operators under consideration.

ad 4)

This is trivial by applying 2) and 3 ).

The quality of the well-posedness we obtained through the refinement of the topologies can be quantified by calculating the pseudoconditionnumber (cf. Nashed and Wahba(1974)) of the inverse problem (8). Here we have

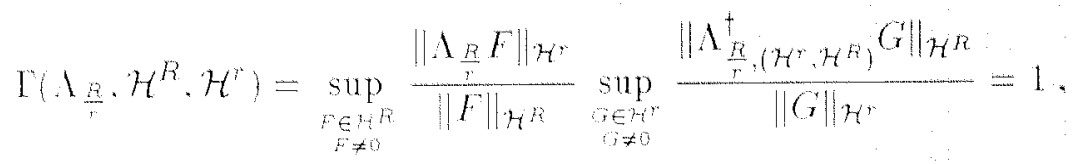

i.e. optimality. Now we have built up the theoretical background for a solution of the inverse problem (8). So in the next section we can proceed with a numerical realization in terms of spherical spline interpolation.

\section{Spherical Spline Interpolation}

In this section we turn over to the problem of calculating a solution of the inverse problem (8) from a function $G \in \mathcal{H}$ given at discrete points $x_{1} \ldots x_{N} \in \Omega_{r}$.

Definition 1 Let $X_{V}^{r}=\left\{x_{1} \ldots x_{N}\right\} \subset \Omega_{r}$ be a set of $N$ distinct points. Any function $S \in \mathcal{H}^{r}$ of the form

$$
S(x)=\sum_{i=1}^{N} a_{i} K_{H}\left(x_{i}, x\right):
$$

$a_{i} \in \mathbb{R}, x \in \Omega_{r}$ is called a spherical spline in $\mathcal{H}^{r}$ relative to $X_{v}$. The class of all spherical splines is denoted by spline $\left.\left\{B_{n}^{n}\right\} . X_{N}\right)$.

The algorithm we want to implement is now as follows:

\section{1. step:}

Given $Y$ values $G\left(r_{i}\right)=G ;: \quad i=1 \ldots \ldots$ of a function $G \in H^{\prime}$. 
Determine $S_{G}^{N} \in S$ pline $\left.\left\{B_{n}^{r}\right\}, X_{N}^{r}\right)$ from $S_{G}^{N}\left(x_{i}\right)=G_{i}, \quad i=1, \ldots, N$.

\section{2. step:}

Define $S_{F}^{N}=\Lambda_{\frac{R}{r},\left(\mathcal{H}^{r}, \mathcal{H}^{R}\right)}^{\dagger} S_{G}^{N}$ as an approximation of $F=\Lambda_{\frac{R}{r},\left(\mathcal{H}^{r}, \mathcal{H}^{R}\right)}^{\dagger} G$ (the desired solution of $(8))$.

For our further investigations we need some basic results of spherical spline interpolation (cf. Freeden(1990)).

1. Uniqueness:

As $B_{n}^{r}>0 ; n=0,1, \ldots$, the interpolation constraints lead to a system of linear equations with positive definite matrix. Thus, there exists a unique solution $S_{G}^{N} \in \operatorname{Spline}\left(\left\{B_{n}^{r}\right\}, X_{N}^{r}\right)$ of the interpolation problem in $\mathcal{H}^{r}$ (1. step).

2. Minimum Norm Properties:

Due to the reproducing property of $K_{H}(\cdot, \cdot), S_{G}^{N}$ satisfies

$$
\inf _{S \in J_{N}(G)}\|S\|_{\mathcal{H}^{r}}=\left\|S_{G r}^{N}\right\|_{\mathcal{H}^{r}}
$$

and

$$
\inf _{\text {SESpline }\left(\left\{B_{n}^{r}\right\}, X_{N}^{+*}\right)}\|S-G\|_{\mathcal{H}^{r}}=\left\|S_{G}^{N}-G\right\|_{\mathcal{H}^{r}}
$$

with

$$
J_{N}(G)=\left\{H \in \mathcal{H}^{r} \mid H\left(x_{i}\right)=G_{i} ; \quad i=1, \ldots, N\right\} .
$$

The next question to be examined is the following: To what extend can these properties be transferred to $S_{F}^{N}$ ?

Uniqueness is trivial as $\Lambda_{\frac{R}{r}}: \mathcal{H}^{R} \rightarrow \mathcal{H}^{r}$ defines an isomorphism. Due to the isometry of $\Lambda_{\frac{R}{r}}$ we additionally have

$$
\left\|S_{F}^{N}\right\|_{\mathcal{H}^{R}} \stackrel{i s o m}{=}\left\|S_{G^{\prime}}^{N}\right\|_{\mathcal{H}^{r}} \stackrel{(9)}{\leq}\|G\|_{\mathcal{H}^{+}} \stackrel{i s o m}{=}\|F\|_{\mathcal{H}^{R}}
$$

and

$$
\left\|S_{F}^{N}-\left.F\right|_{\mathcal{H}^{R}} \stackrel{i s o m}{=}\right\| S_{G}^{N}-G\left\|_{\mathcal{H}^{r}} \stackrel{(10)}{\leq}\right\| S-G\left\|_{\mathcal{H}^{r}} \stackrel{i s o m}{=}\right\| \Lambda_{\frac{R}{r},\left(\mathcal{H}^{r}, \mathcal{H}^{R}\right)}^{\dagger} S-F \|_{\mathcal{H}^{R}}
$$

for all $S \in$ Spline $\left.\left\{B_{n}^{r}\right\}, X_{N}^{r}\right)$. (11) shows the minimal norm character also of the approximation $S_{F}^{N}$, whereas (12) has to be examined more precisely. Let us assume for a moment that $F$ is known on $X_{N}^{R}=\left\{y_{1}, \ldots, y_{N}\right\} \subset \Omega_{R} ; y_{k}=\frac{R}{r} x_{k}$ for $k=1 \ldots N$ (i.e. the projection of $X_{N}^{r}$ onto $\Omega_{R}$ ). Then we solve the interpolation problem

$$
\overline{S_{F}^{N}}\left(y_{i}\right)=F\left(y_{i}\right) ; \quad i=1, \ldots N
$$

in $\left(\tilde{\mathcal{H}},\|\cdot\|_{\tilde{\mathcal{H}}}\right)$. Similarly to $(10)$ we have

$$
\left\|\widetilde{S_{F}^{N}}-F\right\|_{\widetilde{\mathcal{H}}} \leq\|\widetilde{S}-F\|_{\tilde{\mathcal{H}}}
$$




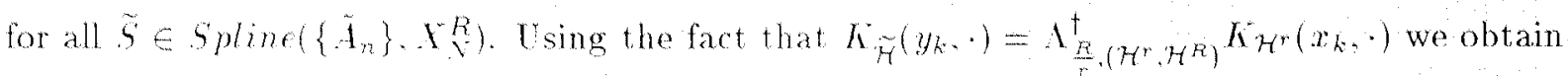
$\operatorname{Spline}\left(\left\{\tilde{A}_{n}\right\}, X_{N}^{R}\right)=\Lambda_{R}^{\dagger}\left(\mathcal{H}^{r}, \mathcal{H}^{R}\right)\left(S p l i n e\left(\left\{B_{n}^{r}\right\}, X_{N}\right)\right)$. Thus. $S_{F}^{N}$ as well as the interpolant $\overline{S_{F}^{N}}$ minimize the "distance" to the function $F$ in the same set of trial functions but with respect to different norms. As $\lim _{n \rightarrow \infty} \sigma_{n}=0$ for all considered SPDO's $\lambda_{n}$ we always have $\widetilde{\mathcal{H}} \subset \mathcal{H}^{R}$ (cf. Theorem 2$)$ and in addition $\|\cdot\|_{\mathcal{H}^{R}} \leq c\|\cdot\|_{\overrightarrow{\mathcal{H}}}(c=$ const $>0)$. For the special case that $\Lambda_{\frac{R}{r}}$ is a SPDO with $. \wedge(n)=1$ for all $n=0,1 \ldots($ cf. Section 2$)$ we even obtain

$$
\left\|S_{F}^{N}-F\right\|_{\mathcal{H}^{R}} \leq\left\|\overline{S_{F}^{N}}-F\right\|_{\breve{\mathcal{H}}} \text {. }
$$

Finally, we want to show convergence which we formulate in

Theorem 3 Given the inverse problem (8) with continuously known righthand side $G \in \mathcal{H}^{r}$, i.e. if $X_{\mathrm{N}}^{*}=\left\{x_{1} \ldots, x_{\mathrm{N}}\right\} \subset \Omega_{r}, x_{k}=r \eta_{k}: k=1 \ldots N$ denotes a set of pairwisely distinct elements such that $X_{X}^{r}=\lim _{N-\infty} X_{\mathrm{N}}$ is dense in $\Omega_{r} . G\left(x_{i}\right)=G_{i} ; i=1, \ldots, N$ is given for any $N \in N$. Then there exists for all $\epsilon>0$ an $N=N(\epsilon) \in N$ and an interpolating spline $S_{G}^{N} \in \operatorname{Spline}\left(\left\{B_{n}^{r}\right\}, X_{Y}\right)$ such that

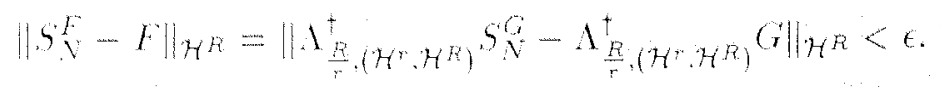

\section{Proof:}

As $\Lambda_{\underline{R}}: H^{R} \rightarrow \mathcal{H}$ is an isometry we only have to show: For any $\epsilon>0$ there exists an $N \in \mathbb{N}$ such that

$$
\left\|S_{V}-F\right\|_{H_{R}}=\left\|S_{\mathrm{V}}^{G}-G\right\|_{H^{\prime}}<\epsilon .
$$

$\Lambda$ s $\mathcal{H}^{r} \subset \mathcal{C}\left(\Omega_{r}\right)$ the set $\left\{\mathcal{H}^{\prime}\left(x_{k}, \cdot\right)\right\}$ is complete and closed in $\mathcal{H}^{r}$, i.e. for all $\epsilon>0$ there exists an $N=N(\epsilon) \in N$ and coefficients $a_{1}, \ldots a_{N} \in \mathbb{R}$ such that

$$
\left\|C_{r}-\sum_{n=1}^{V} a_{n} h_{K^{r}}\left(x_{n} \cdot\right)\right\|_{\mathcal{H}^{r}}<\epsilon
$$

Now we consider the spline $S_{G}^{N} \in S p l i n e\left(\left\{B_{n}^{r}\right\}, X_{N}\right)$ satisfying

$$
S_{G}^{N}\left(x_{k}\right)=G\left(x_{k}\right) ; \quad k=1 \ldots \ldots N .
$$

Due to $(10)$ we have

$$
\left\|S_{G}^{N}-G\right\| H^{r} \leq\|S-G\|_{\mathcal{H}^{r}}
$$

for all $S \in \operatorname{Spline}\left(\left\{B_{n}^{r}\right\}, X_{n}^{r}\right)$. As $S_{G}^{N}$ has the representation

$$
S_{i}^{V}(x)=\sum_{n=1}^{N} b_{n} h H_{n}\left(x_{n} \cdot\right)
$$

with $b_{1} \ldots b_{y}$ being the solution of the linear system of equations due to the interpolation constraints we obtain

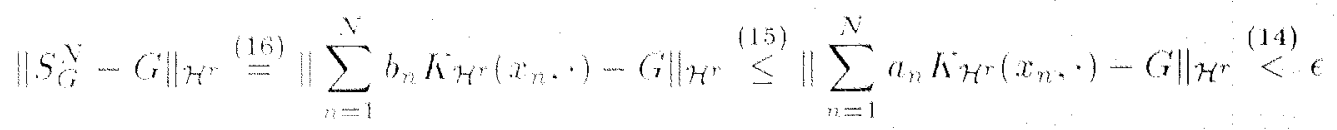

An immediate consequence is 
Corollary 1 Under the same conditions as in Theorem 3 we have

$$
\sup _{y \in \Omega_{R}}\left|S_{F}^{N}(y)-F(y)\right| \stackrel{N \rightarrow \infty}{=} 0 .
$$

\section{Proof:}

We use Theorem 3 togcther with

$$
\begin{aligned}
\left|S_{F}^{N}(y)-F(y)\right| & =\left|\left(S_{F}^{N}-F, K_{\mathcal{H}^{R}}(y, \cdot)\right)_{\mathcal{H}^{R}}\right| \\
& \stackrel{C . S .}{\leq}\left\|S_{F}^{N}-F\right\|_{\mathcal{H}^{R}} \| K_{\mathcal{H}^{R}(y, \cdot) \|_{\mathcal{H}^{R}}} \\
= & \left\|S_{F}^{N}-F\right\|_{\mathcal{H}^{R}} \underbrace{\frac{1}{R^{2}} \sum_{n=0}^{\infty} \frac{2 n+1}{4 \pi} \frac{1}{\left|A_{n}^{R}\right|^{2}}}_{<\infty} .
\end{aligned}
$$

The last result shows the main outcome of the considered algorithm:

We need a certain amount of information only at the satellite orbit to obtain an accurate approximation of the potential on the surface of the spherical earth. This approximation can be determined by the solution of a system of linear equations with positive definite matrix for which powerful solvers are available (e.g. Cholesky decomposition).

So far we considered only inverse problems with error free righthand side. In the following we omit this assumtion and turn over to the solution of inverse problems with noisy input. We consider again the inverse problem (8) but in this case with error affected righthand side $G^{\epsilon} \in \mathcal{H}^{r}$

$$
\Lambda_{\frac{R}{r}} F^{\epsilon}=G^{\epsilon} ; \quad F^{\epsilon} \in \mathcal{H}^{R} .
$$

With strict interpolation (following the previous method) we obtain an interpolant $S_{G^{r}}^{N}$ of the noisy data on satellite altitude and an approximation $S_{F^{c}}^{N}$ of the disturbed solution $F^{\epsilon}$. Instead of doing so we turn over to combined interpolation and smoothing (cf. Freeden and Witte(1982)).

Given the set $X_{N}^{r}=\left\{x_{1}, \ldots x_{N}\right\} \subset \Omega_{r}$, the values $G^{\epsilon}\left(x_{i}\right)=G_{i}^{\epsilon} ; i=1, \ldots, N$ and prescribed positive weights $\delta, \gamma_{1}^{2}, \ldots, \gamma_{N}^{2}$, such that the matrix

$$
\left(K_{H} \mathcal{H}^{r}\left(x_{i}, x_{j}\right)+\delta \gamma_{i}^{2} \delta_{i j}\right) \underset{\substack{i=1, \ldots, N \\ j=1 \ldots, N}}{N}
$$

is regular. Then

$$
S_{G^{*}}^{\delta_{\gamma}}(x)=\sum_{i=1}^{N} a_{i} K_{K^{r}}\left(x_{i}, x\right)
$$

with coefficients $a_{1} \ldots \ldots a_{x}$ uniquely determined by

$$
S_{G}^{\delta}\left(x_{i}\right)+\delta \gamma_{i}^{2} a_{i}-G_{i}^{\epsilon}
$$


satisfies

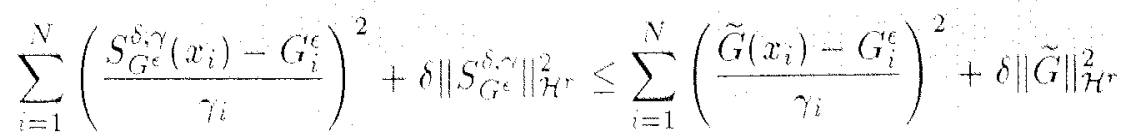

for all $\tilde{G} \in \mathcal{H}^{r}$. The first part of this functional controls the distance of the spline to the given function at the points $r_{1} \ldots \ldots r$. while the second part controls the smoothness of the spline. Now we examine again to what extend the approximation $S_{V^{\gamma} \gamma}^{\phi}=\Lambda_{\frac{H}{r},\left(\mathcal{H}^{r}, \mathcal{H}^{R}\right)}^{\dagger} S_{G^{t}}^{\delta_{\gamma} \gamma}$ satisfies adequate minimizing conditions. By applying Theorem 2 the following result is easily verified

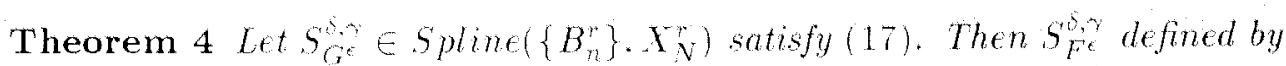

$$
S_{F c}^{\beta, \gamma}(y)=\left(\Lambda_{\frac{R}{r},\left(H^{\prime}, H^{R}\right)}^{\dagger} S_{G^{c}}^{S}\right)(y)
$$

satisfies

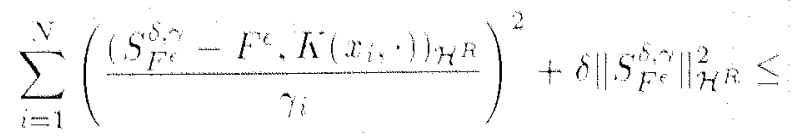

$$
\begin{aligned}
& \sum_{i=1}^{v}\left(\frac{\left(\tilde{F}-F^{t} \cdot k\left(x_{i} \cdot\right)\right)_{H^{R}}}{\gamma_{i}}\right)^{2}+\delta\|\tilde{F}\|_{H^{R}}^{2}
\end{aligned}
$$

for all $\tilde{F} \in \mathcal{H}^{R}$ and

$$
h(x, y)=\sum_{n=0}^{\infty} \sum_{j=1}^{2 n+1} f\left(\sigma_{n}\right) \sigma_{n}^{-1} Y_{n, j}^{r}(x) Y_{n, j}^{R}(y)
$$

This functional also consists of two parts. Here, the first part controls the distance between a function of class $\mathcal{H}^{R}$ and the disturbed solution of the inverse problem in a $(\cdot, \cdot)_{\mathcal{H}^{R} \text {-inner product }}$ sense, while the second part controls again the smoothness of the function. The choice of the parameters $\gamma i$ and $\delta$ is the critical point in this approach. It sounds reasonable to adapt them to the standart deviations of the measured values. while in the special case that $\gamma_{i}=\sqrt{N}$ we refer to Wahba(1990), where the methods of ordinary and generalized cross validation are presented to obtain good values for $\delta$ from the given data $C_{i}$.

Until now we always assumed the spherical shape of the orbit as well as the spherical shape of the surface of the earth. In the next section we turn over to more general geometries. With this we are able to deal with realistic satellite missions. 


\section{The Generalization to Non-Spherical Structures}

\subsection{The New Geometries and the Corresponding Hilbert Spaces}

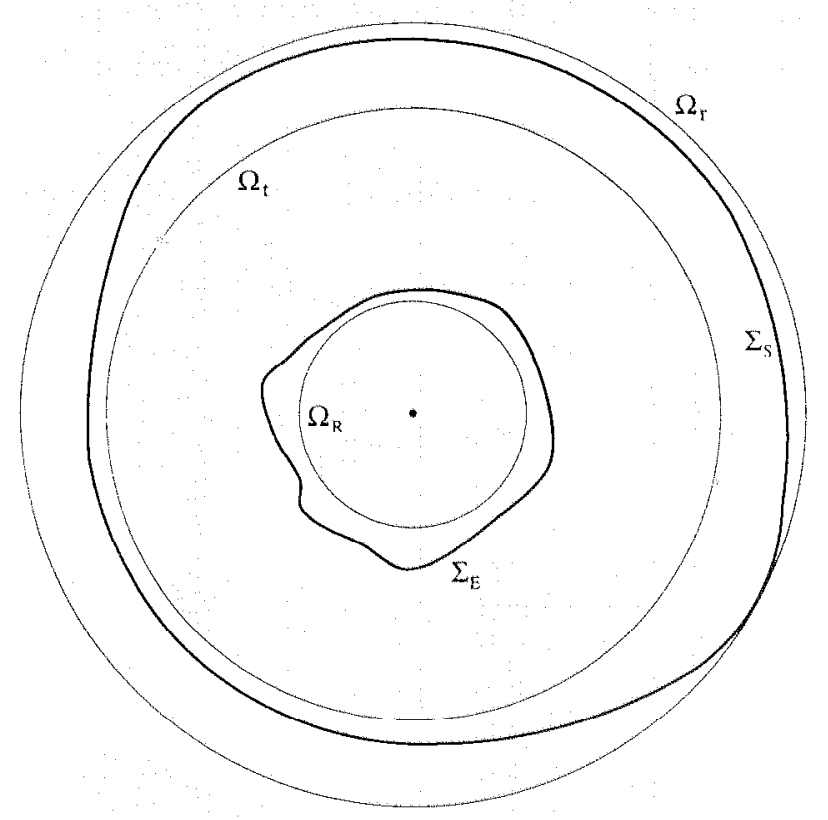

Figure 1

According to the real world conditions we consider the following geometry, where $\Sigma_{E}$ denotes the surface of the earth and $\Sigma_{S}$ a compact surface situated in the cxtcrior of a sphere $\Omega_{t}$, i.e. $t<\inf _{x \in \Sigma_{S}}|x|$, where the data is measured. $\Sigma_{S}$ has the properties that it divides $\mathbb{R}^{3}$ into the bounded region $\Sigma_{S}^{i n t}$ (containing the origin) and the unbounded region $\Sigma_{S}^{e x t}$ and that any radius starting from the origin intersects $\Sigma_{S}$ only once. For $\Sigma_{E}$ we only require that it is a compact surface contained in the exterior of a Bjerhammar sphere $\Omega_{R}$, i.e. $R$ has to satisfy $R<\inf _{x \in \Sigma_{E}}|x|<t$. Finally, the sphere $\Omega_{r}$ is defined by $r=\sup _{x \in \Sigma_{S}}|x|$ (see Figure 1).

Firstly, we want to mention that $\Omega_{R}$ and $\Omega_{r}$ do not denote the spherical earth and the spherical satellite orbit as in the previous sections. Here they denote an inscribing sphere of the earth and a covering sphere of the orbit. Nevertheless we use the same notation as we want to work with the same spaces already introduced in Section 4.

Let $\mathcal{H}^{R} \subset \mathcal{C}\left(\Omega_{R}\right)$ be defined as in Theorem 1. Then for all $F \in \mathcal{H}^{R}$.

$$
\mathcal{F}(z)=\int_{\Omega_{R}} A_{\frac{R}{|z|}}(y, z) F(y) d \omega_{R}(y)
$$

$|z|>R$ with

$$
A_{|=|}(y, z)=\sum_{n=0}^{\infty} \sum_{j=1}^{2 n+1}\left(\frac{R}{|z|}\right)^{n} Y_{n, j}^{R}(y) Y_{n, j}^{|z|}(z)
$$


represents the solution of the first boundary value problem of potential theory (exterior Dirichlet problem) with respect to the sphere $\Omega_{n}$ (cf. e.g. Kellogg(1967)). Thus, the functions

$$
\mathcal{F}(z)=\sum_{n=0}^{\infty} \sum_{j=1}^{2 n+1}\left(\frac{R}{|z|}\right)^{n} F_{n, j}^{R} Y_{n, j}^{|z|}(z)
$$

form a linear space denoted by $\mathcal{H}_{B}(\mathrm{~B}=\mathrm{Below})$. On $\mathcal{H}_{B}$ we define an inner product $(\cdot, \cdot)_{\mathcal{H}_{B}}$ as follows. Let $\mathcal{F}_{1}, \mathcal{F}_{2}$ be of class $\mathcal{H}_{B}$. Then we set

$$
\left(\mathcal{F}_{1}, \mathcal{F}_{2}\right)_{\mathcal{H}_{B}}=\left(F_{1}, F_{2}\right)_{H^{R}}
$$

where $F_{1}$ and $F_{2}$ denote the functions of class $H^{R}$ such that

$$
\mathcal{F}_{i}(z)=\int_{\Omega_{R}} A_{\frac{R}{|\lambda|}}(y, z) F_{i}(y) d \omega_{R}(y): i=1,2
$$

$|*|>R$. The inner product is well defined as the linear operator $A_{\frac{R}{R}}: \mathcal{H}^{R} \rightarrow \mathcal{H}_{B} ; F \mapsto \mathcal{F}$ is isomorphic. Then the corresponding norm is defined as usual by

$$
\|\mathcal{F}\|_{\mathcal{H}_{B}}=\left((\mathcal{F}, \mathcal{F})_{\mathcal{H}_{B}}\right)^{\frac{1}{2}} \text {. }
$$

Hence, $\mathcal{H}_{B}$ equipped with the inner product $(19)$ is a separable Hilbert space of "harmonic continuations" from functions in $\mathcal{H}^{R}$ to $\Omega_{R}^{c x t}=\left\{x \in \mathbb{R}^{3}|| x \mid>R\right\}$. Therefore we immediately obtain $H_{B} \subset \mathcal{C}^{\infty}\left(\Omega_{R}^{*}\right)$.

\section{Lemma 2}

$$
K_{\mathcal{H}_{B}}(\tilde{z} \cdot \tilde{z})=\sum_{n=0}^{\infty} \sum_{j=1}^{2 n+1}\left(\frac{R}{|z|}\right)^{n}\left(\frac{R}{|z|}\right)^{n} f\left(\sigma_{n}\right) \sigma_{n}^{-2} Y_{n, j}^{|\theta|}(z) Y_{n, j}^{|z|}(z)
$$

$|z|,|\tilde{z}|>R$ is the reproducing kernel in $\mathcal{H}_{B}$.

\section{Proof:}

Straightforward using (19).

As we are lateron interested in the solution of our problem at the surface of the earth $\Sigma_{E}$ we define the restriction operator $P_{\mathbb{S}_{E}}$ by

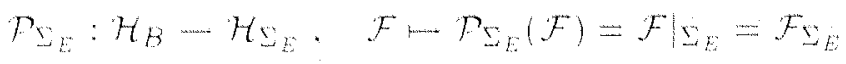

which is the restriction of $J \in H_{B}$ onto the surface $\Sigma_{E}$.

$$
H_{\Sigma_{E}}=\left\{F_{\Sigma_{E}} \mid \mathcal{F}_{\Sigma_{E}}=P_{\Sigma_{E}}(F): \mathcal{F} \in \mathcal{H}_{B}\right\} \subset C^{\infty}\left(\Sigma_{E}\right)
$$

denotes the space of all restrictions. In a similar way we equip $\mathcal{H}_{\mathrm{S}_{B}}$ with the inner product $(\cdot)_{Z_{E}}$, defined by

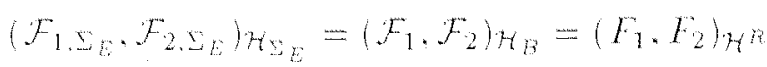


and the corresponding norm

$$
\left\|\mathcal{F}_{\Sigma_{E}}\right\|_{\mathcal{H}_{\Sigma_{E}}}=\left(\left(\mathcal{F}_{\Sigma_{E}}, \mathcal{F}_{\Sigma_{E}}\right)_{\mathcal{H}_{\Sigma_{E}}}\right)^{\frac{1}{2}}
$$

As $\Sigma_{E}$ is a compact surface in $\Omega_{R}^{e x t}$, any function $\mathcal{F} \in \mathcal{H}_{B}$ is uniquely determined by its restriction onto $\mathcal{H}_{\Sigma_{E}}$. Thus, $\mathcal{P}_{\Sigma_{E}}$ defines as well an isometric isomorphism between $\mathcal{H}_{B}$ and $\mathcal{H}_{\Sigma_{E}}$. By equivalent means we define now the spaces at satellite altitude $(A=$ Above $)$.

$$
\mathcal{H}_{A}=\left\{\mathcal{G} \mid \mathcal{G}(x)=\int_{\Omega_{t}} A_{t|t|}(x, w) G(w) d \omega_{t}(w) ; G \in \mathcal{H}^{t}\right\}
$$

$|x|>t$, where $\mathcal{H}^{t}=\mathcal{H}\left(\left\{C_{n}^{t}\right\}, \Omega_{t}\right)$ and $C_{n}^{t}=\left(f\left(\sigma_{n}\right)\left(\frac{r}{t}\right)^{2 n}\right)^{-\frac{1}{2}}$. Due to the conditions on $f$ (see Theorem 1) it can be easily calculated that $\left\{C_{n}^{t}\right\}$ is summable and thus $\mathcal{H}^{t} \subset \mathcal{C}\left(\Omega_{t}\right)$ defines a reproducing kernel Hilbert space. On $\mathcal{H}_{A}$ we define the inner product

$$
\left(\mathcal{G}_{1}, \mathcal{G}_{2}\right)_{\mathcal{H}_{A}}=\left(G_{1}, G_{2}\right)_{\mathcal{H}^{t}}
$$

and the associated norm

$$
\left\|\mathcal{G}_{1}\right\|_{\mathcal{H}_{A}}=\left(\left(\mathcal{G}_{1}, \mathcal{G}_{1}\right)_{\mathcal{H}_{A}}\right)^{\frac{1}{2}}
$$

where $\mathcal{G}_{1}, \mathcal{G}_{2}$ are defined by

$$
\mathcal{G}_{i}(x)=\int_{\Omega_{\mathrm{t}}} A_{\frac{\ell}{|x|}}(x, w) G_{i}(w) d \omega_{t}(w): i=1.2
$$

$|x|>t$. Thus, $\left(\mathcal{H}_{A},(\cdot, \cdot)_{\mathcal{H}_{A}}\right)$ forms a separable Hilbert space and $\mathcal{H}_{A} \subset \mathcal{C}^{\infty}\left(\Omega_{t}^{e x t}\right)$.

\section{Lemma 3}

$$
\bar{L}_{\mathcal{H}_{A}}(\widehat{x}, x)=\sum_{n=0}^{\infty} \sum_{j=1}^{2 n+1}\left(\frac{r}{|\widehat{x}|}\right)^{n}\left(\frac{r}{|x|}\right)^{n} f\left(\sigma_{n}\right) Y_{n, j}^{|\widehat{x}|}(\widehat{x}) Y_{n, j}^{|x|}(x)
$$

$|x|,|\widehat{x}|>t$ is the reproducing kernel in $\mathcal{H}_{A}$.

\section{Proof:}

In analogy to Lemma 2.

Furthermore we obtain

Lemma 4 Let $G^{r}(x)=\left.\mathcal{G}(x)\right|_{|x|=r}$ denote the restriction of $\mathcal{G} \in \mathcal{H}_{A}$ onto $\Omega_{r}$. Then $\left\|G^{r}\right\|_{\mathcal{H}^{r}}=\|\mathcal{G}\|_{\mathcal{H}_{A}}$.

\section{Proof:}

Let $\mathcal{G}$ be of class $\mathcal{H}_{A}$. Thus.

$$
\mathcal{G}(\hat{x})=\sum_{n=0}^{\infty} \sum_{j=1}^{2 n+1}\left(\frac{t}{|\hat{x}|}\right)^{n} G_{n, j}^{t} Y_{n, j}^{F|\hat{x}|}(\hat{x})
$$


$|x|>t$. Then

$$
\| G_{H_{i}}^{2}=\sum_{n=0}^{\infty} \sum_{l=1}^{2 n+1}\left(f\left(\sigma_{n}\right)\right)^{-1}\left(\frac{t}{r}\right)^{2 n}\left(G_{n, j}^{t}\right)^{2}
$$

Moreover, we have

$$
G^{n}(x)=\sum_{n=0}^{\infty} \sum_{j=1}^{2 n+1}\left(\frac{t}{r}\right)^{n} G_{n, j}^{t} Y_{n, j}^{r}(x)
$$

$|x|=r$ and

$$
\left\|G^{r}\right\|_{\mathcal{H}^{r}}^{2}=\sum_{n=0}^{\infty} \sum_{j=1}^{2 n+1}\left(f\left(\sigma_{n}\right)\right)^{-1}\left(\frac{t}{r}\right)^{2 n}\left(G_{n, j}\right)^{2}
$$

In the same way as before we define $P_{-}$by

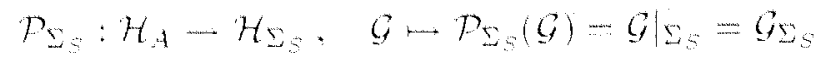

and

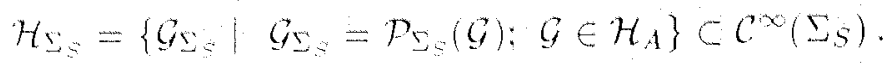

Hence $H_{5}$ equipped with the inner product $(\cdot)_{\infty}$ defined by

$$
\left(G_{1}, S_{S}, G_{2} \Sigma_{S}\right) H_{S}=\left(G_{1}, G_{2}\right) H_{A}=\left(G_{1},\left(G_{2}\right) H_{H}\right.
$$

is a reproducing kernel Hilbert space.

Now we have finished the preparations we need to proced with the inverse problem (8) with respect to more general geometric settings.

\subsection{The Generalization of the Inverse Problem}

Let us assume for a moment that we are given the following inverse problems

$$
\left(\Lambda_{\frac{R}{12}} F_{u}\right)(x)=G^{32}(x)
$$

$|x|=u$ for all $t<u \leq r$ with $F_{u} \in \mathcal{H}^{R}, C_{i}^{n} \in \mathcal{H}^{u}=\mathcal{H}\left(\left\{C_{n}\right\}, \Omega_{u}\right), C_{n}=\left(\int\left(\sigma_{n}\right)\left(\frac{r}{u}\right)^{2 n}\right)^{-\frac{1}{2}}$ and

$$
\left(\Lambda_{\frac{R}{n}} f\right)(x)=\int_{\Omega R}\left(\sum_{n=0}^{x} \sum_{j=1}^{2 n+1}\left(\frac{R}{u}\right)^{n} \wedge \wedge(n) Y_{n, j}^{R}(y) Y_{n, j}^{n}(x)\right) F(y) d \omega_{R}(y)
$$

i.e. the righthand side is given for any sphere $\Omega_{z}$ betweon $\Omega_{R}$ and $\Omega_{r}$. Next we consider an arbitrary but fixed $u \in(t, r)$. As $A_{\frac{i z}{r}}: \mathcal{H}^{u}-\mathcal{H}^{r}$ defined by

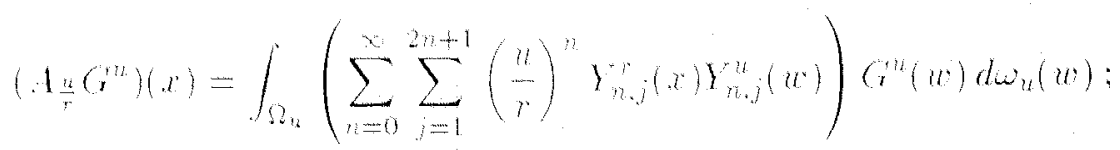


$|x|=r$ denotes an isometric isomorphism between $\mathcal{H}^{n}$ and $\mathcal{H}^{r}$ (see Lemma 4 ), the unique solution of $(20)$ is given by

$$
F_{u}(y)=\left(\Lambda_{\frac{R}{r},\left(\mathcal{H}^{r}, \mathcal{H}^{R}\right)}^{\dagger}\left(A_{\frac{u}{r}} G^{u}\right)\right)(y) .
$$

For $u=r$ we simply have

$$
F_{r}(y)=\left(\Lambda_{\frac{R}{r},\left(\mathcal{H}^{r}, \mathcal{H}^{R}\right)}^{\dagger} G^{r}\right)(y) \text {. }
$$

If we define $G^{u}(x)=\left.\mathcal{G}(x)\right|_{|x|=u}, \mathcal{G} \in \mathcal{H}_{A}$ for all $t<u \leq r$ we consequently have $F_{u}=F_{r}$ for all $u \in(t, r]$ as all restrictions $G^{u}$ lead to the same function $G^{r}$. Under these assumptions we consider the inverse problem

$$
\left(\Lambda_{\Sigma_{E}, \Sigma_{S}} \mathcal{F}_{\Sigma_{E}}\right)(x)=\mathcal{G}_{\Sigma_{S}}(x)
$$

$x \in \Sigma_{S}, \mathcal{G}_{\Sigma_{S}} \in \mathcal{H}_{\Sigma_{S}}$ and $\mathcal{F}_{\Sigma_{E}} \in \mathcal{H}_{\Sigma_{E}}$, where $\Lambda_{\Sigma_{E}, \Sigma_{S}}$ is defined by

$$
\left(\Lambda_{\Sigma_{E}, \Sigma_{S}} \mathcal{F}_{\Sigma_{E}}\right)(x)=\left(\Lambda_{\frac{R}{|x|}} F\right)(x)
$$

and $F \in \mathcal{H}^{R}$ given by (18). The operator is well defined because of the requirements on $\Sigma_{S}$. Due to the isometric isomorphism between $\mathcal{H}_{\Sigma_{S}}$ and $\mathcal{H}^{r}$ (via $\mathcal{P}_{\Sigma_{S}}$ and Lemma 4), $\mathcal{G}_{\Sigma_{S}}$ defines a unique function $G^{r} \in \mathcal{H}^{r}$ and the mique solution of $(21)$ is given by

$$
\mathcal{F}_{\Sigma_{E}}(z)=\mathcal{P}_{\Sigma_{E}}\left(\int_{\Omega_{R}} A_{\frac{R}{|z|}}(y, z) F(y) d \omega_{R}(y)\right)
$$

with

$$
F(y)=\left(\Lambda_{\frac{R}{r},\left(\mathcal{H}^{r}, \mathcal{H}^{R}\right)}^{\dagger} G^{r}\right)(y)
$$

Finally, Figure? shows the different steps of the solution of (?1)

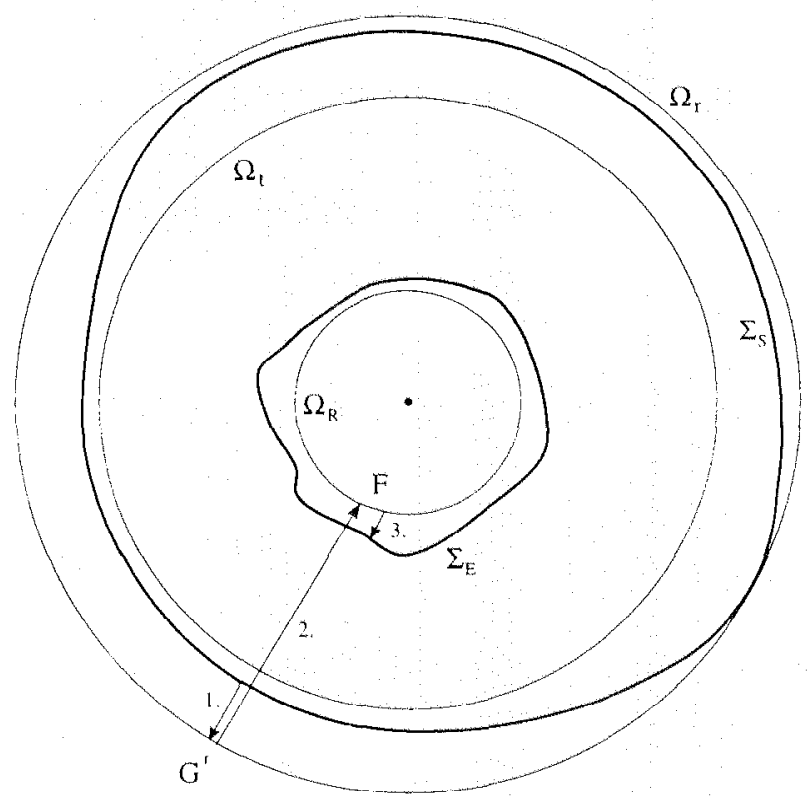

Figure 2 


\section{$6.3 H_{4}$ - Spline Interpolation}

In this section we generalize the spherical spline interpolation of Section 5 with respect to the inverse problem (21). Given a function $\mathcal{G} \in \mathcal{H}_{A}$ at discrete points $x_{1}, \ldots, x_{N} \in \mathbb{\Xi}_{S} \subset \mathbb{R}^{3}$ with $t<\left|x_{i}\right| \leq r$ for $i=1 \ldots, v$ we look for an approximation of the unique solution $\mathcal{F}_{\Sigma_{E}}(z)$ given by $(22)$. In contrast to the ordinary spherical spline interpolation we have to require regularity for the matrix in the linear system of equations due to the interpolation constraints (cf. Freeden(1981) and Freeden(1987)).

Definition 2 The set $X_{N}=\left\{x_{1} \ldots x_{N}\right\} \subset\left(\Omega_{t}^{e x t} \cap \Omega_{\gamma}^{i n t}\right)$ is called $H_{A}$-fundamental system if the $N \times N$-matrix

$$
\Gamma=\left(\begin{array}{lll}
\kappa_{\mathcal{H}_{A}}\left(x_{1}, x_{1}\right) & \cdots & K_{\mathcal{H}_{A}}\left(x_{1}, x_{N}\right) \\
\vdots & & \vdots \\
K_{\mathcal{H}_{A}}\left(x_{N}, x_{1}\right) & \cdots & K_{\mathcal{H}_{A}}\left(x_{N}, x_{N}\right)
\end{array}\right)
$$

is regular.

This enables us to define a $\mathcal{H},-$ spline.

Definition 3 Let $X_{N}$ be a $\mathcal{H}_{A}$-fundamental system. Then any function $S \in \mathcal{H}_{A}$ of the form

$$
\mathcal{S}(x)=\sum_{i=1}^{N} a_{i} K_{\mathcal{H}_{A}}\left(x_{i}, x\right) ;
$$

$a_{i} \in \mathbb{R}, t<|x| \leq r$ is called a $\mathcal{H}_{A}$-spline relative to $X_{N}$. The class of all $\mathcal{H}_{A}$-splines is denoted by Spline $\left(\mathcal{H}_{A}, X_{N}\right)$.

If $X_{N} \subset \Sigma_{S}$ we can also define a $\mathcal{H}_{\Sigma_{S}}$ spline. As $\mathcal{P}_{\Sigma_{S}}\left(\mathcal{H}_{A}\right)=\mathcal{H}_{\Sigma_{S}}$ and $\mathcal{P}_{\Sigma_{S}}\left(K_{\mathcal{H}_{A}}\left(x_{i}, \cdot\right)\right)=$ $K_{\mathcal{H}_{S}}\left(x_{i}, \cdot\right)$ we define $P_{\mathbb{S}_{S}}(\mathcal{S})=\mathcal{S}_{\Sigma_{5}}$ for any $S \in \operatorname{Spline}\left(\mathcal{H}_{A}, X_{N}\right)$ and

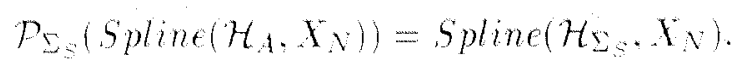

The algorithm we want to implement can now be described as follows:

\section{1. step:}

Given a $H_{4}$-fundamental svetem $\mathrm{S}_{\mathrm{V}}=\left\{x_{1} \ldots x_{\mathrm{V}}\right\} \subset \mathrm{S}_{\mathrm{S}}$ and discrete values $\mathcal{G}\left(x_{i}\right)=$ $\mathcal{G}_{\Sigma_{S}}\left(x_{i}\right)=\mathcal{G}_{i}: i=1 \ldots \ldots$ of a function $G_{B} \in H_{A}$

Determine $S_{G}^{N} \in S p l i n e\left(\mathcal{H}_{1}, X_{N}\right)$ from $S_{G}^{V}\left(x_{i}\right)-G_{i}: i=1 \ldots N$.

2. step:

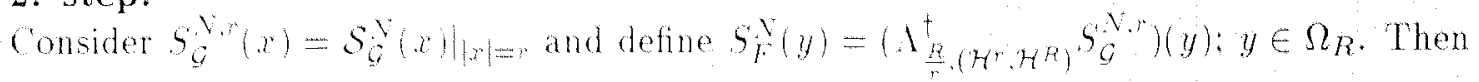

$$
S_{F}^{N}(z)=\int_{\Omega_{R}} d_{\frac{R}{R}}(y, z) S_{R}^{N}(y) d \omega_{R}(y)
$$


denotes an approximation of

$$
\mathcal{F}(z)=\int_{\Omega_{R}} A_{\frac{R}{|z|}}(y, z) F(y) d \omega_{R}(y)
$$

where $F=\Lambda_{\frac{R}{r},\left(\mathcal{H}^{r}, \mathcal{H}^{R}\right)}^{\dagger} G^{r}$. Finally, $\mathcal{S}_{\mathcal{F}_{\Sigma_{E}}^{N}}^{N}=\mathcal{P}_{\Sigma_{E}}\left(\mathcal{S}_{\mathcal{F}}^{N}\right)$ is an approximation of the solution of the inverse problem (21). In analogy to the ordinary spherical spline interpolation we have

1. Uniqueness:

A unique $\mathcal{H}_{A^{-}}$spline interpolant $\mathcal{S}_{G}^{N}$ is guaranteed by the requirements on $X_{N}^{\Sigma_{S}}$ while the uniqueness of $S_{F}^{N}, \mathcal{S}_{\mathcal{F}}^{N}$ and $\mathcal{S}_{\mathcal{F}_{\Sigma_{E}}}^{N}$ is obtained by the algorithm.

\section{Minimum Norm Properties}

As in the ordinary spherical spline interpolation case we have

$$
\inf _{\mathcal{S} \in J_{N}(G)}\|\mathcal{S}\|_{\mathcal{H}_{A}}=\left\|\mathcal{S}_{\mathcal{C}}^{N}\right\|_{\mathcal{H}_{A}}
$$

and

$$
\inf _{\operatorname{SESpline}\left(\mathcal{H}_{A}, X_{N}^{\Sigma S}\right)}\|\mathcal{S}-\mathcal{G}\|_{\mathcal{H}_{A}}=\left\|\mathcal{S}_{\mathcal{G}}^{N}-\mathcal{G}\right\|_{\mathcal{H}_{A}}
$$

with

$$
J_{N}(\mathcal{G})=\left\{\tilde{\mathcal{G}} \in \mathcal{H}_{A} \mid \tilde{\mathcal{G}}\left(x_{i}\right)=\mathcal{G}_{i} ; \quad i-1, \ldots, N\right\} .
$$

Next we examine the minimal norm properties of $S_{F}^{N}, S_{\mathcal{F}}^{N}$ and $\mathcal{S}_{\mathcal{F}_{\mathcal{F}_{t}}^{N}}$, respectively.

$$
\begin{aligned}
& \left\|\mathcal{S}_{\mathcal{F}_{\Sigma_{E}}}^{N}\right\|_{\mathcal{H}_{\Sigma_{E}}}=\left\|\mathcal{S}_{\mathcal{F}}^{N}\right\|_{\mathcal{H}_{B}}=\left\|S_{F}^{N}\right\|_{\mathcal{H}^{R}} \stackrel{\Lambda^{\dagger}}{{ }^{\dagger},\left(\mathcal{H}^{r}, \mathcal{H}^{R}\right)^{i s o m .}}\left\|S_{\mathcal{G}}^{N, r}\right\|_{\mathcal{H}^{r}} \stackrel{\text { Lemma }}{=}\left\|\mathcal{S}_{\mathcal{G}}^{N}\right\|_{\mathcal{H}_{A}} \\
& \stackrel{(23)}{\leq}\|\mathcal{G}\|_{\mathcal{H}_{A}} \stackrel{\text { Lemma }}{=}\left\|G^{r}\right\|_{\mathcal{H}^{r}} \stackrel{{ }^{\Lambda_{R}}}{=}=\|F\|_{\mathcal{H}^{R}}=\|\mathcal{F}\|_{\mathcal{H}_{B}}=\left\|\mathcal{F}_{\Sigma_{E}}\right\|_{\mathcal{H}_{\Sigma_{E}}}
\end{aligned}
$$

Furthermore, we obtain

$$
\begin{gathered}
\left\|\mathcal{S}_{\mathcal{F}_{\Sigma_{E}}^{N}}^{N}-\mathcal{F}_{\Sigma_{E}}\right\|_{\mathcal{H}_{\Sigma_{E}}}=\left\|\mathcal{S}_{\mathcal{F}}^{N}-\mathcal{F}\right\|_{\mathcal{H}_{B}}=\left\|S_{F}^{N}-F\right\|_{\mathcal{H}^{R}}=\left\|\mathcal{S}_{G}^{N}-\mathcal{G}\right\|_{\mathcal{H}_{A}} \stackrel{(24)}{\leq} \\
\|\mathcal{S}-\mathcal{G}\|_{\mathcal{H}_{A}}=\left\|S^{N, r}-G^{r}\right\|_{\mathcal{H}^{r}}=\left\|\Lambda_{\frac{R}{r},\left(\mathcal{H}^{r}, \mathcal{H}^{R}\right)}^{\dagger} S^{N, r}-F\right\|_{\mathcal{H}^{R}}= \\
\left\|\int_{\Omega_{R}} A_{\frac{R}{I}}(y, \cdot)\left(\Lambda_{\frac{R}{r},\left(\mathcal{H}^{r}, \mathcal{H}^{R}\right)}^{\dagger} S^{N, r}\right)(y) d \omega_{R}(y)-\mathcal{F}\right\|_{\mathcal{H}_{B}}= \\
\left\|\mathcal{P}_{\Sigma_{E}}\left(\int_{\Omega_{R}} A_{R}(y \cdot)\left(\Lambda_{\frac{R}{r}\left(\mathcal{H}^{r}, \mathcal{H}^{R}\right)}^{\dagger} S^{N, r}\right)(y) d \omega_{R}(y)\right)-\mathcal{F}_{\Sigma_{E}}\right\|_{\mathcal{H}_{\Sigma_{E}}}
\end{gathered}
$$

with

$$
S^{N, r}(x)=\sum_{i=1}^{N} b_{i} \sum_{n=0}^{\infty} \sum_{j=1}^{2 n+1}\left(\frac{r}{u_{i}}\right)^{n} f\left(\sigma_{n}\right) Y_{n, j}^{u_{i}}\left(x_{i}\right) Y_{n, j}^{r}(x)
$$

$\left|x_{i}\right|=u_{i} ; i=1 \ldots \ldots$ and arbitrary $b_{1} \ldots \ldots b_{N} \in \mathbb{R} .(25)$ shows again the minimal norm character of the approximations $\mathcal{S}_{\mathcal{F}}^{X}$ and $\mathcal{S}_{\mathcal{F}_{S_{E}}}^{N}$ with respect to $\|\cdot\|_{\mathcal{H}_{B}}$ and $\|\cdot\|_{\mathcal{H}_{\Sigma_{S}}}$. respectively. Calculating

$$
\widetilde{\mathcal{S}}(z)=\left(\int_{\Omega_{R}} A_{\frac{R}{|=|}}(y, z)\left(\Lambda_{\frac{R}{r},\left(\mathcal{H}^{r}, \mathcal{H}^{R}\right)}^{\dagger} S^{N, r}\right)(y) d \omega_{R}(y)\right.
$$


we obtain

$$
\mathcal{S}(z)=\sum_{i=1}^{N} b_{i} H_{(}\left(x_{i}, z\right)
$$

with $\widetilde{K}\left(x_{i} \cdot\right) \in \mathcal{H}_{B}$ defined by

$$
\widetilde{K}\left(x_{i}, z\right)-\sum_{n=0}^{\infty} \sum_{j=1}^{2 n+1}\left(\frac{r}{u_{i}}\right)^{n}\left(\frac{R}{|z|}\right)^{n} f\left(\sigma_{n}\right) \sigma_{n}^{-1} Y_{n, j}^{n}\left(x_{i}\right) Y_{n, j}^{|z|}(z) .
$$

Thus, inequality (26) points out the minimization of the "distance" between the approximation $\mathcal{S}_{F}^{N}$ and the unknown function $\mathcal{F}$ in the reference set $\left\{\widetilde{K}\left(x_{1}, \cdot\right) \ldots \widetilde{K}\left(x_{N}, \cdot\right)\right\} \subset \mathcal{H}_{B}$. Finally, we have to show convergence which we state in

Theorem 5 Given the inverse problem (21) with righthand side $\mathcal{G}_{\mathrm{S}_{5}} \in \mathcal{H}_{\mathrm{S}_{5}}$ which is assumed to be continuously known on $\Sigma_{s}$. i.e. if $X_{V}^{S}=\left\{x_{1}, \ldots, x_{N}\right\} \subset \Sigma_{s}, x_{2}=u_{i} \eta_{i}, \eta_{i} \in \Omega_{1}$ for $i=$ $1, \ldots, N$ denotes a $\mathcal{H}_{A}$-fundamental system such that $\lim _{N \rightarrow \infty} X_{N}$ is dense in $\Sigma_{S}, \mathcal{G}_{\Sigma_{S}}\left(x_{i}\right)=$ $\mathcal{G}_{i}: i=1, \ldots, N$ is given for any $N \in N$. Then for all $\epsilon>0$ there exists an $N=N(\epsilon) \in N$ and an interpolating spline $\mathcal{S}_{\mathcal{G}_{-}}^{N} \in \operatorname{Spline}\left(\mathcal{H}_{\Sigma_{3}}, X_{N}^{E}\right)$ such that

$$
\left\|S_{Y_{E}}-F_{Y_{E}}\right\|_{\mathcal{H}_{Z}}<\epsilon \text {. }
$$

\section{Proof:}

$$
\begin{aligned}
& \left\|\mathcal{S}_{\mathcal{F}_{E}}-\mathcal{F}_{\mathcal{E}_{E}}\right\|_{\mathcal{H}_{E_{B}}}=\left\|\mathcal{S}_{\mathcal{F}}^{\mathrm{V}}-\mathcal{F}_{\mathcal{H}_{B}}=\right\| S_{F}^{\mathrm{V}}-F \|_{\mathcal{H}^{R}}
\end{aligned}
$$

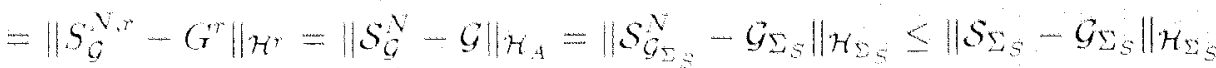

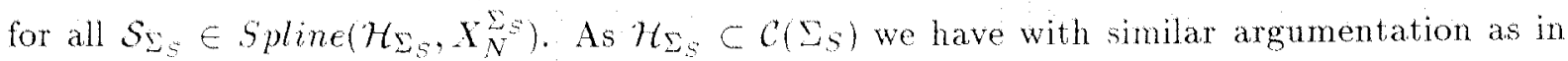
Theorem 3

$$
\left\|\mathcal{S}_{\mathcal{F}_{\Sigma_{E}}}^{N}-\mathcal{F}_{\Sigma_{E}}\right\|_{\mathcal{H}_{\Sigma_{E}}}<\epsilon
$$

In other words: If the data at satellite altitude becomes denser the approximation of the desired solution $\mathcal{F}_{\Sigma_{E}}$ converges at the surface of the earth to the unknown potential. Again we immediately obtain

Corollary 2 Under the same conditions as in Theorem 5 we have

$$
\operatorname{sip}_{z \in E_{E}}\left|S_{E_{E}}(z)-F_{E_{E}}(\tilde{z})\right|^{\mathrm{N}-\infty} 0
$$

But here we can also show that $S_{x}^{V}$ converges to $\mathcal{F}$ in the whole exterior of the earth.

Theorem 6 lnder the same conditions as in theorem 5 we have

$$
\sup _{z \in \Sigma_{E}}\left|S_{E}(z)-F(z)\right|^{Y-\infty} 0
$$




\section{Proof:}

Let $\approx \in \Sigma_{E}^{e x t}$. Then

$$
\begin{aligned}
\left|\mathcal{S}_{\mathcal{F}}^{N}(z)-\mathcal{F}(z)\right| & =\left|\left(\mathcal{S}_{\mathcal{F}}^{N}-\mathcal{F}, K_{\mathcal{H}_{B}}(z \cdot)\right)_{\mathcal{H}_{B}}\right| \\
& \leq\left\|\mathcal{S}_{\mathcal{F}}^{N}-\mathcal{F}\right\|_{\mathcal{H}_{B}}\left\|K_{\mathcal{H}_{B}}(z, \cdot)\right\|_{\mathcal{H}_{B}} \\
& =\left\|\mathcal{S}_{\mathcal{F}_{\Sigma_{E}}}^{N}-\mathcal{F}_{\Sigma_{E}}\right\|_{\mathcal{H}_{\Sigma_{E}}} \frac{1}{|z|^{2}} \sum_{n=0}^{\infty} \frac{2 n+1}{4 \pi} f\left(\sigma_{n}\right) \sigma_{n}^{-2}\left(\frac{R}{|z|}\right)^{2 n} \\
& \leq \underbrace{\left\|\mathcal{S}_{\mathcal{F}_{\Sigma_{E}}}^{N}-\mathcal{F}_{\Sigma_{E}}\right\| \mathcal{H}_{\Sigma_{E}}}_{<\epsilon} \underbrace{\frac{1}{R^{2}} \sum_{n=0}^{\infty} \frac{2 n+1}{4 \pi} f\left(\sigma_{n}\right) \sigma_{n}^{-2}}_{<\infty}
\end{aligned}
$$

independent of $z$.

Finally, we want to mention that also in this case a combined interpolation and smoothing result similarly to the spherical approach can be obtained.

\subsection{Explicit Sequences}

The critical point in our approach is the choice of the function $f$ such that $\left(f\left(\sigma_{n}\right)\left(\frac{r}{R}\right)^{2 n}\right)^{-\frac{1}{2}}$ is summable (see Theorem 1). Candidates may be found among the regularizing filters (cf. e.g. Louis(1989)). Examples are the iterated filters of Tikhonov regularization

$$
f\left(\sigma_{n}\right)=\frac{\sigma_{n}^{k}}{\left(\sigma_{n}^{2}+\gamma^{2}\right)^{\frac{k}{2}}} \gamma>0, k \geq 3
$$

Thus,

$$
\begin{aligned}
\sum_{n=0}^{\infty} \frac{2 n+1}{4 \pi} \frac{\sigma_{n}^{k}}{\left(\sigma_{n}^{2}+\gamma^{2}\right)^{\frac{k}{2}}}\left(\frac{r}{R}\right)^{2 n} & =\sum_{n=0}^{\infty} \frac{2 n+1}{4 \pi} \frac{\left(\frac{R}{r}\right)^{k n}\left(\Lambda^{\wedge}(n)\right)^{k}}{\left(\left(\left(\frac{R}{r}\right)^{n} \Lambda^{\wedge}(n)\right)^{2}+\gamma^{2}\right)^{\frac{k}{2}}}\left(\frac{r}{R}\right)^{2 n} \\
& =\sum_{n=0}^{\infty} \frac{2 n+1}{4 \pi} \frac{\left(\frac{R}{r}\right)^{(k-2) n}\left(\Lambda^{\wedge}(n)\right)^{k}}{\left(\left(\left(\frac{R}{r}\right)^{n} \Lambda^{\wedge}(n)\right)^{2}+\gamma^{2}\right)^{\frac{k}{2}}} \\
& <\infty
\end{aligned}
$$

as $\Lambda$ is a SPDO of order $t<x$. Another admissible choice is $f\left(\sigma_{n}\right)=s^{n}\left(\frac{R}{r}\right)^{2 n} \Lambda^{\wedge}(n)$, $0<|s|<1$ which leads obviously to a summable sequence $\left(f\left(\sigma_{n}\right)\left(\frac{r}{R}\right)^{2 n}\right)^{-\frac{1}{2}}$. Morcover, it is possible to represent all occuring reproducing kernels as elementary functions for any permitted $\Lambda^{\wedge}(n)$. 


\subsection{Numerical Example}

In this section we calculate the solution of

$$
\left(\log _{F_{B}} F_{D_{E}}\right)(x)=G_{S}(x)
$$

for the following input (long measure: $1 \mathrm{~km}$ ):

1. The orbit is assumed to be contained in the surface of the ellipsoid

$$
\Sigma_{S}=\left\{x \in \mathbb{R}^{3} \mid \frac{x_{1}^{2}}{6574^{2}}+\frac{x_{2}^{2}}{6574^{2}}+\frac{x_{3}^{2}}{6555^{2}}=1\right\}
$$

with $\Omega_{t}=\Omega_{6554}$ and $\Omega_{r}=\Omega_{6.574}$.

2. The earth is assumed to be approximated by

$$
S_{E}=\left\{x \in \mathbb{R}^{3} \mid \frac{x_{1}^{2}}{6378^{2}}+\frac{x_{2}^{2}}{6378^{2}}+\frac{x_{3}^{2}}{6356^{2}}=1\right\}
$$

with $\Omega_{R}=\Omega_{6355}$ (i.e. the mean altitude of the orbit is about $200 \mathrm{~km}$ ).

3. As righthandside $G_{S}$ we take the artificial function visualized in Figure 3

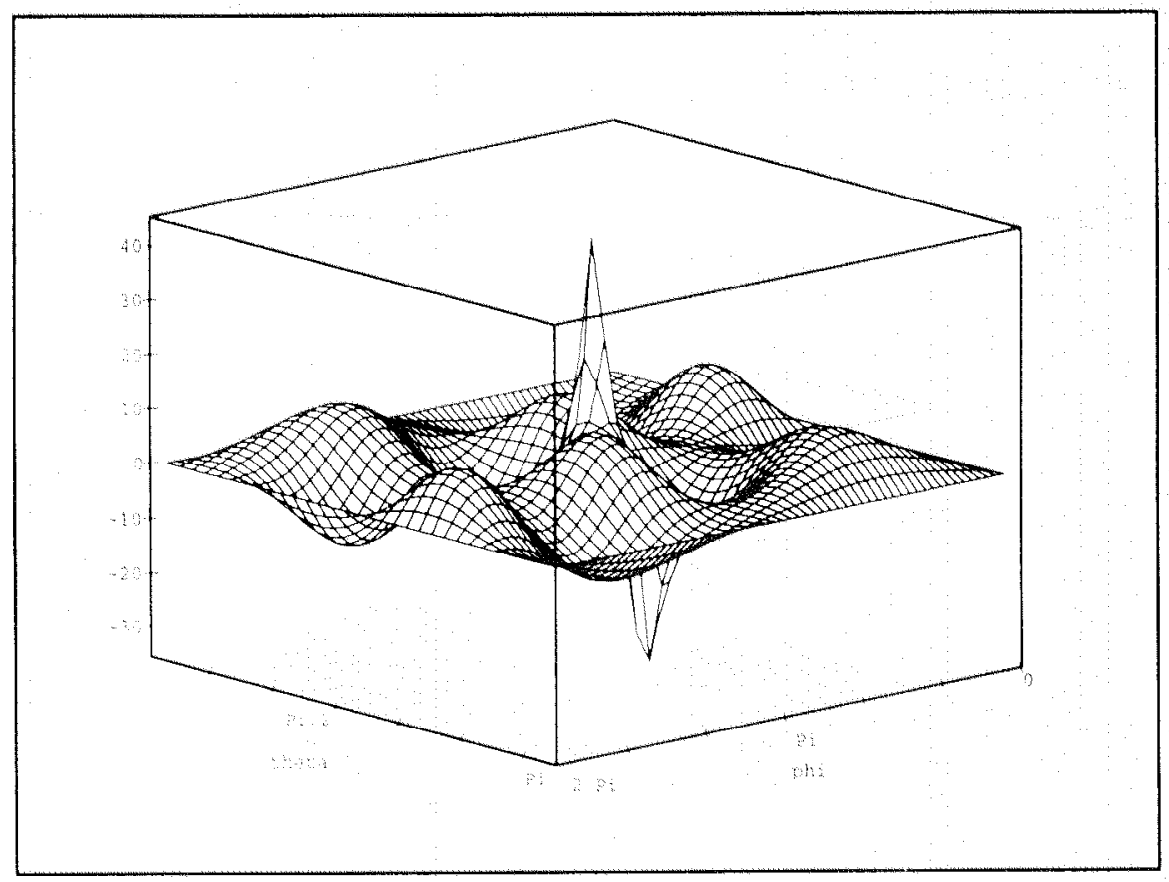

Figure 3

which is assumed to be given at 2011 points on 5.5 . 
4. We choose the sequence $f\left(\sigma_{n}\right)$ as

$$
f\left(\sigma_{n}\right)=s^{n}\left(\frac{R}{r}\right)^{2 n} \Lambda^{\wedge}(n)
$$

with $s=0.9$ and $\Lambda^{\wedge}(n)=1$. Using the fact that

$$
\sum_{n=0}^{\infty} s^{n} P_{n}(t)=\frac{1}{\left(1+s^{2}-2 s t\right)^{\frac{1}{2}}}
$$

(cf. e.g. Müller(1966)), we obtain.

$$
K_{H_{A}}(x, \widehat{x})=\frac{1}{|x||\widehat{x}| 4 \pi} \frac{1-\left(\frac{s R^{2}}{|x||x|}\right)^{2}}{\left(1+\left(\frac{s R^{2}}{|x||\widehat{x}|}\right)^{2}-2 \frac{s R^{2}}{\left.|x||x| \frac{x}{|x|} \frac{\widehat{x}}{|\widehat{x}|}\right)^{\frac{3}{2}}}\right.}
$$

and

$$
\int_{\Omega_{R}} A_{\frac{R}{|z|}}(y, z)\left(\left.\Lambda_{\frac{R}{r},\left(\mathcal{H}^{r}, \mathcal{H}^{R}\right)}^{\dagger} K_{\mathcal{H}_{A}}(x, \hat{x})\right|_{|x|=r}\right)(y) d \omega_{R}(y)=\frac{1}{|z||\widehat{x}| 4 \pi} \frac{1-\left(\frac{s|z|}{|x|}\right)^{2}}{\left(1+\left(\frac{s|z|}{|x|}\right)^{2}-2 \frac{s|z|}{\left.|x| \frac{z}{|z|} \frac{\widehat{x}}{|\hat{x}|}\right)^{\frac{3}{2}}}\right.}
$$

Finally, Figure 4 shows the approximation $\mathcal{S}_{\mathcal{F}_{\Sigma_{E}}}^{N}$ of the solution $\mathcal{F}_{\Sigma_{E}}$ on $\Sigma_{E}$ and the absolute error $\left|\mathcal{S}_{\mathcal{F}_{\Sigma_{E}}}^{N}(x)-\mathcal{F}_{\Sigma_{E}}(x)\right|$, for $x \in\left\{x_{1}, \ldots, x_{1 \tau 40}\right\} \subset \Sigma_{E}$, is illustrated in Figure 5 .

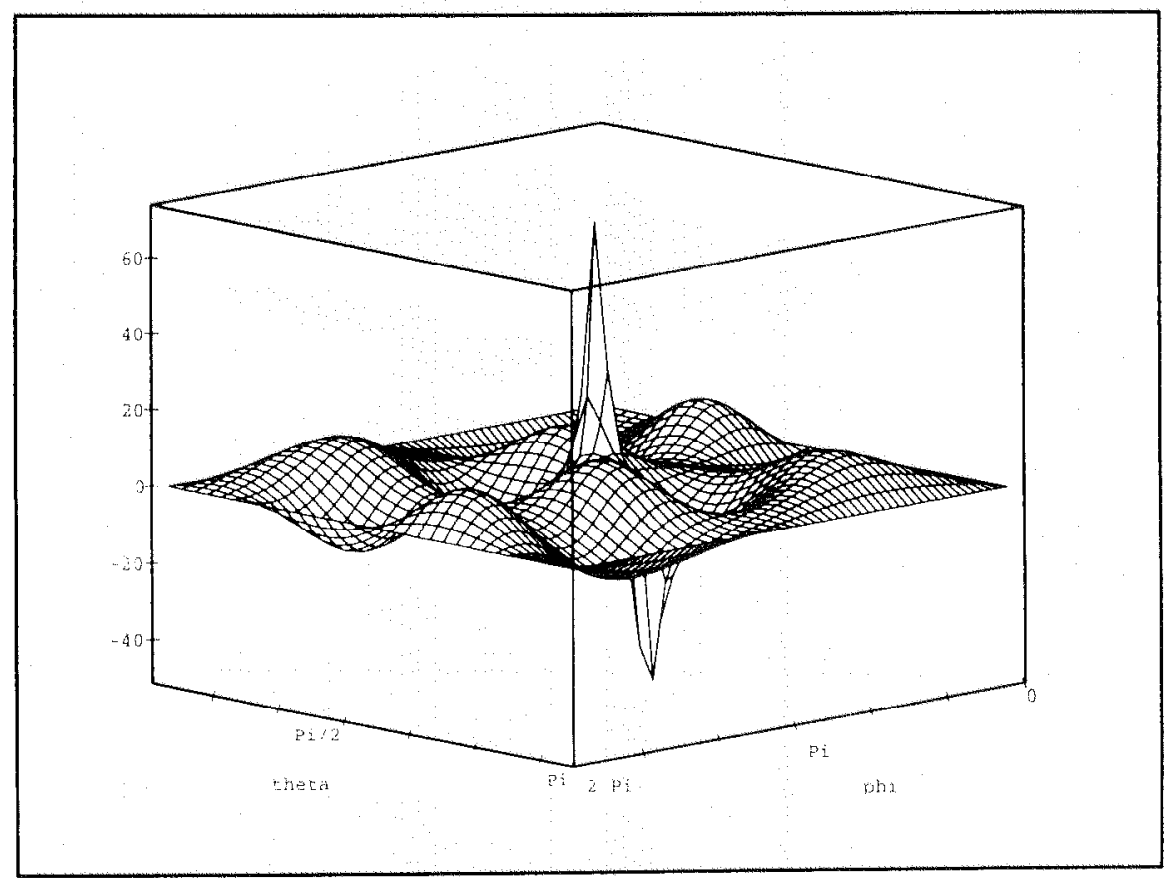

Figure 4 


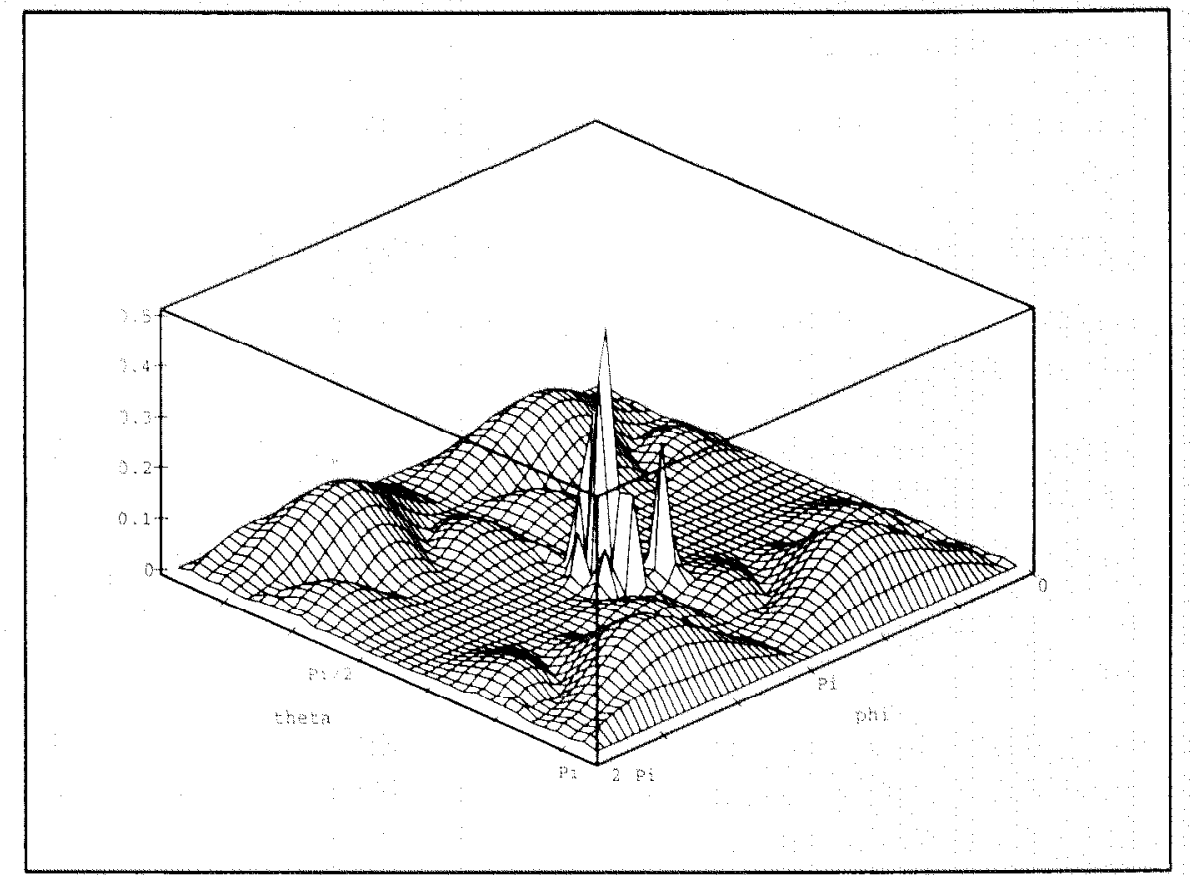

Figure 5

\section{References}

Aronszajn, N. (1950): Theory of Reproducing Kernels, Trans. Am. Wath. Soc. 68, 337-404

Cui, J. and Freeden, W. (1995): Equidistribution on the Sphere, AGTM-Report 142, University of Kaiserslautern. Geomathematics Group

Ėskin, G.I. (1981): Boundary Value Problems for Elliptic Pseudodifferential Equations, Transl. of Math. Monogr. 52 American Math. Soc. Providence. Rhodt Island

Freeden, W. (1979): Über eine Klasse von Integralformeln der Mathematischen Geodäsie, Vorff. Gcod. Inst. RWTH Aachen. Heft 27

Freeden, W. (1981): On Approximation by Harmonic Splines, Momuscr. Geod. 6, 193-244

Freeden, W. (1987): A Spline Interpolation Method for Solving Boundary Value Problems of Potential Theory from Discretely Given Data. Numer, Meth. of Partial Diff. Eq. 3, $375-398$

Freeden, W. (1990): Spherical Spline Approximation and Its Application in Phisical Geodesy, in: Geophysical Data Inersion Wethods and Applications (A. Vogel. C.O. Ofeagbu, R. Gorenfo. B. Ersin ets). Tieweg Publication. 79-104

Freeden, W. and Witte, B. (1982): \& Combined (Spline-) Interpolation and Smoothing Method for the Determination of the Lxternal Gravitational Potential from Heterogeneous Data. Bull. Geod. 56. 5360? 
Keller, W. and IIirsch, M. (1994): A Boundary Value Approach to Downward Continuation. Manuscr. Geod. 2. 101-118

Kellogg, O.D. (1967): Foundations of Potential Theory, Springer-Verlag Berlin Heidelberg Now York

Louis, A.K. (1989): Inverse und schlecht gestellte Probleme, R.G. Teubner Stuttgart

Meschkowski, H. (1962): Hilbertsche Räume mit Kernfunktionen, Springer-Verlag Berlin Göttingen Heidelberg

Müller, C. (1966): Spherical Harmonics, Lecture Notes in Mathematics, 17, Springer-Verlag, Berlin, Heidelberg, New York

Nashed, M.Z. and Wahba, G. (1974): Generalized Inverses in Reproducing Kernel Spaces: An Approach to Regularization of Linear Operator Equations, SIAM J. Math. Anal., Vol 5. No. 6. $974-987$

Rummel, R. (1979): Determination of Short-Wavelength Components of the Gravity Field from Satellite-to-Satellite Tracking or Satellite Gradiometry - An Attempt to an Identification of Problem Areas, Manuscr. Geod. 4. 107-148

Rummel, R. (1986): Satellite Gradiometry, in: Sünkel, H. ed. ,Lecture Notes in Earth Sciences 7, Mathematical and Numerical Techniques in Physical Geodesy, Springer, Berlin, $318-363$

Schreiner, M. (1994): Tensor Spherical Harmonics and Their Application in Satellite Gradiometry, Doctoral-Thesis. University of Kaiserslautern, Geomathematics Group

Svensson, L. (1984): Finite Elements on the Sphere, Journal of Approx. Theory 40 , Academic Press, $246-260$

Thalhammer, M. (1995): Regionale Gravitationsfeldbestimmung mit zukünftigen Satellitenmissionen (SST und Gradiometry), Deutsche Geodätische Kommission bei der Bayerischen Akademie der Wissenschaften. Reihe C. Dissertationen, Heft Nr. 437

Wahba, G. (1973): Convergence Rates of Certain Approximate Solutions to Fredholm Integral Equations of the First Kind, J. of Approx. Theory 7, 167-185

Wahba,G. (1990): Spline Models for Observational Data, CBMS-NSF Regional Conference Series in Applied Mathematics 59 